
\title{
$\begin{array}{ll}\text { Research Square } & \begin{array}{l}\text { Preprints are preliminary reports that have not undergone peer review. } \\ \text { They should not be considered conclusive, used to inform clinical practice, } \\ \text { or referenced by the media as validated information. }\end{array}\end{array}$
}

\section{Early alterations of circulating immune cell counts in severe trauma patients are related to later occurrence of nosocomial infection, sepsis and mortality}

\section{Xijie Dong}

Tongji Hospital of Tongji Medical College of Huazhong University of Science and Technology

\section{Chuntao Wang}

Tongji Hospital of Tongji Medical College of Huazhong University of Science and Technology

Xinghua Liu

Tongji Hospital of Tongji Medical College of Huazhong University of Science and Technology

Xiangjun Bai

Tongji Hospital of Tongji Medical College of Huazhong University of Science and Technology

Zhanfei Li ( $\square$ whtj_lzf@163.com )

https://orcid.org/0000-0001-5058-6052

\section{Research}

Keywords: trauma, injury severity score, neutrophil count, lymphocyte count, monocyte count, neutrophil-lymphocyte ratio, nosocomial infection, sepsis, mortality

Posted Date: December 23rd, 2019

DOI: https://doi.org/10.21203/rs.2.19574/v1

License: (c) (i) This work is licensed under a Creative Commons Attribution 4.0 International License. Read Full License 


\section{Abstract}

Background: Severe trauma leads to extensive disturbances of the innate and adaptive arms of the immune system, which in turn may affect the prognosis. The main objective of this study was to investigate the relationship between the alterations of circulating immune cell counts in the early stage of severe trauma and the later occurrence of nosocomial infection, sepsis and mortality.

Methods: This was a retrospective study of 876 patients with an Injury Severity Score (ISS) $\geq 16$. Demographic data, the absolute counts of neutrophil, lymphocyte and monocyte (ANC, ALC and AMC) on days 1, 3, and 7 (D1, D3, and D7) after trauma, and whether nosocomial infection, sepsis or death occurred within 60 days were recorded. Ratios were calculated between immune cell counts of each two time points, namely day 3/day 1 (D3/D1) and day 7/day 3 (D7/D3). Patients were grouped based on ISS and the occurrence of nosocomial infection, sepsis or death. Comparative studies were conducted between each two groups. Univariate and multivariate logistic regression analysis were used to identify variables related to the risk of nosocomial infection, sepsis, and mortality. Receiver operating characteristic (ROC) curve was plotted to assess the predictive value of various risk factors.

Results: More severe trauma leads to more pronounced increase in ANC and more slowly recovery of ALC. In patients with subsequent nosocomial infection and sepsis, ANC was higher and ALC recovery was slower than those without nosocomial infection and sepsis within 7 days. In non-survivors, ALC had not recovered and AMC (D3) and AMC (D7) was lower than survivors within 7 days. ALC (D3) and ALC (D3/D1) are independent risk factors for nosocomial infection and sepsis. ALC (D3), ALC (D3/D1), ALC (D7) and AMC (D7) are independent risk factors for death. The combination of ALC (D3/D1) and ALC (D3) had a good predictive value for the occurrence of nosocomial infection, sepsis, and mortality.

Conclusions: More severe trauma causes more intense interference to circulating immunocyte counts. Worse alterations in circulating immunocyte counts within 7 days may increase the risk of subsequent nosocomial infection, sepsis and mortality.

\section{Background}

Trauma is a disease with high morbidity and mortality. More than five million people die of trauma every year. It is also the primary cause of death in individuals under 40 years old [1,2]. The main cause of early death at the scene or after admission is mainly severe brain damage or massive bleeding due to heart or large blood vessel injury. With the advancement of resuscitation strategies, damage control surgery and supportive care, many inpatients can survive the early stage. However, for these early survivors, delayed death due to sepsis and multiple organ dysfunction syndrome (MODS) is also frequent [3-6].

Extensive tissue injury and ischemia-induced release of damage-related molecular models (DAMP) after severe trauma leads to a tremendous inflammatory response (systemic inflammatory response syndrome, SIRS) and an extremely important antiinflammatory response (compensatory anti-inflammatory response syndrome, CARS) occurs almost simultaneously [4, 7-10]. These early events are generally believed to disrupt the homeostasis of the immune system and are important causes of nosocomial infection, sepsis and MODS in the later stage [11-14]. And this disruption of homeostasis is quite complex, affecting the innate and adaptive arms of the immune system, leading to significant changes in the count, function and phenotype of the immune cells $[4,15,16]$. Nevertheless, if we continue to systematically deepen the study of these immune cell changes, we may also have the opportunity to find out general rules in the complexity. This in turn may help us to predict that some patients may have adverse complications or poor prognosis in advance, for which we can conduct early intervention. Moreover, the changes of these immune cells may also be the cause of adverse complications or poor prognosis, which may also help us to expand the idea of immunotherapy.

Many studies have previously reported some changes in the post-traumatic immune system and the impact of these changes on prognosis. However, the following points may be worth further consideration: Simultaneously studying the response of innate and adaptive immune cells in the same group of patients to post-traumatic immune system status is more comprehensive than studying one type of immune cells alone; Considering that the immune system is dynamic, it is more persuasive to study the law of change in a period of time than to investigate the state of a time point; Although some of the predictive test indicators are very meaningful, they are not routinely used clinically, more easily available and repeatable indicators need to be introduced. 
In this study, we collected a large number of target patients and used a common clinical test item, blood routine, to comprehensively study the alterations in the absolute counts of neutrophil, lymphocyte and monocyte (ANC, ALC and AMC) of severe trauma patients within 7 days. They cover most of the innate and adaptive immune cells. In addition, we evaluated the predictive value of these immune cell counts at certain time points and their changes over time for the risk of nosocomial infections, sepsis and death within 60 days.

\section{Methods}

Study design, setting and patients

This retrospective study was performed at a 7,000-bed university-affiliated hospital. Approval was obtained from the Medical Ethics Committee of the hospital with a waiver of informed consent.

Patients admitted within 24 hours after trauma with an Injury Severity Score (ISS) $\geq 16$ were enrolled in this study. The exclusion criteria were patients less than 18 years old; those with autoimmune or hypersensitivity diseases; neoplastic or proliferative hematologic diseases; severe systemic inflammation caused by other diseases (e.g. liver cirrhosis, myocardial infarction); concurrent chronic disease requiring treatment with any form of immunomodulation therapy. Besides, patients were also excluded if they had non-survivable injuries (ISS $=75$ ) or died within 7 days. This is because our purpose was to investigate the relationship between the alterations of immunocyte counts within 7 days in the early survivors and the later emergence of nosocomial infections, sepsis and death. These patients are not suitable. Furthermore, to minimize the impact of surgery on the immune cell count results, we excluded patients who underwent surgery from after blood collection on day 1 to before blood collection on day 7 .

Data collection

Demographic data, Acute Physiology and Chronic Health Evaluation (APACHE) II score, ISS, Glasgow coma scale (GCS), the mechanism of injury, days of ventilator use, co-morbidities, smoking or not, whether nosocomial infection, sepsis or death occurred within 60 days, and blood routine results (ANC, ALC and AMC) on days 1, 3, and 7 (D1, D3, and D7) were obtained from the clinical information systems. Patients with incomplete information were also withdrawn from this study.

In our study, nosocomial infection refers to bacteremia, respiratory system, urinary system, surgical site, or skin and soft tissue infection occurring after 3 days from admission. Sepsis is defined as life-threatening organ dysfunction caused by a dysregulated host response to infection; organ dysfunction can be identified as an acute change in total SOFA score $\geq 2$ due to infection [17].

\section{Statistical analysis}

Continuous and categorical variables are reported as mean (SD), median (interquartile range [IQR]) and number (proportion) as appropriate. The Kolmogorov-Smirnov test was used to verify the normality of continuous data. Comparisons of three correlated samples were performed using Friedman's rank test (for non-normally distributed data) followed by the Wilcoxon signed rank test. Comparisons of two independent samples were performed using Wilcoxon rank sum test (for non-normally distributed data), MannWhitney $\mathrm{U}$ test (for ordered categorical data) or chi-square tests (for unordered categorical data). Pearson correlation coefficient was used to measure the multicollinearity of continuous variables. Univariate and multivariate logistic regression analysis were used to identify variables related to the risk of nosocomial infection, sepsis, and mortality. Receiver operating characteristic (ROC) curve was plotted to calculate the area under the ROC curve and determine the cut-off value. Binary logistic regression model was used for the combination analysis of two indicators. Statistical analyses and graphics were developed using SPSS v20.0 (IBM, Armonk, NY), MedCalc v19.0.7 (MedCalc Software, Ostend, Belgium) and GraphPad Prism v8.3.0 (GraphPad Software, San Diego, CA). A p value less than 0.05 was considered significant.

\section{Results}

Patients

A total of 876 patients with an ISS of $24.0(19.0,29.0)$ were screened for inclusion into the present study. Table 1 shows baseline characteristics for the entire patient cohort. The incidence of nosocomial infection and sepsis was $54.3 \%$ (476 patients) and $27.2 \%$ 
(238 patients), respectively. The overall mortality rate was $18.4 \%$ (161 patients).

Table 1

Overall characteristics of trauma patients

\begin{tabular}{|c|c|}
\hline Parameter & Patient $(n=876)$ \\
\hline Age, mean (SD), year & $49.8(14.7)$ \\
\hline Male, n (\%) & $194(22.1)$ \\
\hline APACHE II score, median (IQR) & $11.0(7.0,15.0)$ \\
\hline ISS, median (IQR) & $24.0(19.0,29.0)$ \\
\hline \multicolumn{2}{|l|}{ GCS, n (\%) } \\
\hline $3-8$ & $166(18.9)$ \\
\hline $9-12$ & $120(13.7)$ \\
\hline $13-15$ & $590(67.4)$ \\
\hline \multicolumn{2}{|l|}{ Mechanism of injury, n (\%) } \\
\hline Fall & $336(38.4)$ \\
\hline Road traffic collision & $328(37.4)$ \\
\hline Tumble & $151(17.2)$ \\
\hline Assault & $20(2.3)$ \\
\hline Other/unknown & $41(4.7)$ \\
\hline Operated on admission, n (\%) & $187(21.3)$ \\
\hline Ventilator days, median (IQR) & $0(0,4.0)$ \\
\hline \multicolumn{2}{|l|}{ Co-morbidities, n (\%) } \\
\hline Hypertension & $111(12.7)$ \\
\hline Coronary artery disease & $17(1.9)$ \\
\hline Cerebrovascular disease & $18(2.1)$ \\
\hline Diabetes mellitus & $48(5.5)$ \\
\hline COPD & $18(2.1)$ \\
\hline Smoking, n (\%) & $289(33.0)$ \\
\hline Nosocomial infection, n (\%) & $476(54.3)$ \\
\hline Sepsis, n (\%) & $238(27.2)$ \\
\hline 60-day mortality, n (\%) & $161(18.4)$ \\
\hline
\end{tabular}

Severity of trauma

According to ISS, we divided the patients into two groups: $16 \leq$ ISS $\leq 24$ and $25 \leq$ ISS $\leq 74$. The latter has higher APACHE II score, lower GCS and longer ventilator use time (Additional file 1: Table S1). ANC and AMC rise and ALC decline were common in severe trauma patients. The proportions of patients with immune cell counts above the upper limit of the normal range of ANC and AMC 
or below the lower limit of the normal range of ALC are shown on the x-axis (Fig. 1). Patients with higher ISS had higher ANC within 7 days. Although ANC of both groups showed a decrease on the 3rd day, it rebounded in patients with higher ISS on the 7th day (Fig. 1A). On the 1st day, there was no significant difference in ALC between the two groups. Then, ALC of the lower ISS group gradually increased from the 3rd day, while ALC of the higher ISS group began to rise on the 7th day. And ALC of the latter was lower than the former on the 3rd and 7th day (Fig. 1B). Besides, AMC increased in the higher ISS group on the 7th day, but there was no significant difference between the two groups (Fig. 1C). These results show that patients suffering from more severe trauma have worse alterations in immune cell counts, especially ANC and ALC.

Nosocomial infection

According to whether nosocomial infection occurred, patients were divided into two groups: non-nosocomial infection and nosocomial infection. A total of 476 patients had nosocomial infection, with the lungs being the most common site, and Gramnegative bacteria being the most common pathogen (data not shown). Nosocomial infection group had higher ISS and APACHE II score, lower GCS and longer ventilator use time (Additional file 1: Table S1). The trends of differences in ANC and ALC between the non-nosocomial and nosocomial infection groups were similar to those between the lower and the higher ISS groups (Fig. 1A, 1B, $2 \mathrm{~A}$ and $2 \mathrm{~B}$ ). The $\mathrm{AMC}$ of the nosocomial infection group increased on the 7th day, and was higher than the non-nosocomial infection group (Fig. 2C). Besides, given the wide fluctuation of the of immune cell counts, ratios were calculated between their counts of each two time points, namely day 3/day 1 (D3/D1) and day 7/day 3 (D7/D3). ANC (D3/D1), ALC (D3/D1), ANC (D7/D3) and AMC (D7/D3) were different between the two groups (Fig. 2D). These results suggest that subsequent nosocomial infection may be associated with worse alterations of immune cell counts in the early stage.

\section{Sepsis}

According to whether sepsis occurred, we divided the patients into two groups: non-sepsis and sepsis. A total of 238 patients developed sepsis, with the lungs being the most common site of infection, and Gram-negative bacteria being the most common pathogen (data not shown). Sepsis patients were older, with higher ISS and APACHE II score, lower GCS and longer ventilator use time (Additional file 1: Table S1). The trends of differences in ANC and ALC between the non-sepsis and sepsis groups were similar to those between the lower and higher ISS groups or the non-nosocomial infection and nosocomial infection groups (Fig. 1A, 1B, 2A, 2B, 3A and 3B). On day 7, AMC of sepsis group increased and was higher than non-sepsis group (Fig. 3C). Besides, ANC (D3/D1), ALC (D3/D1) and ANC (D7/D3) was different between the two groups (Fig. 3D). These results indicate that worse alterations of immune cell counts may increase the risk of sepsis.

Mortality

According to whether death occurred within 60 days, patients were divided into two groups: alive and death. A total of 161 patients were included in the latter group, and they were older, with higher ISS and APACHE II score, lower GCS, and longer ventilator use time compared with the former group (Additional file 1: Table S1). ANC of both groups reduced on the 3rd day and rebounded on the 7th day, but the latter group was different from the former group in that the ANC increased to the same level as the 1st day on day 7. And there was a difference in ANC between the two groups on day 7 (Fig. 4A). There was no difference in ALC between the two groups on day 1, it gradually increased in the alive group but no significant change in the death group. As a result, ALC differed between the two groups on the 3rd and 7th days (Fig. 4B). Besides, AMC increased on the 7th day in the alive group and was higher than the death group on the 3rd and 7th days (Fig. 4C). Moreover, ALC (D3/D1), AMC (D3/D1) and AMC (D7/D3) were different between the two groups (Fig. 4D). These results suggest that worse alterations in immune cell populations may increase the risk of mortality.

We further divided the non-survivors into two groups according to the cause of death: sepsis and trauma. There were 96 (59.6\%) patients died of sepsis and $65(40.4 \%)$ patients died of trauma. There were no significant differences in age, gender, ISS, APACHE II score, and GCS between the two groups, as well as ANC, ALC, and AMC (Fig. 4E, 4F, 4G and Additional file 1: Table S1). These results imply that regardless of whether the patients eventually died of sepsis or trauma, they may suffer a similar degree and potentially fatal immune system insult in the early stage.

Risk factor analysis

Page 5/22 
To further determine the relationship between the immune cell counts and the occurrence of nosocomial infection, sepsis and mortality, we performed risk factor analysis. Since the median time of nosocomial infection was day 6 (data not shown) in our study, we did not analyze the effect of ANC (D7), ALC (D7) and AMC (D7) on nosocomial infection. The results of univariate and multivariate analysis for immune cell counts at fixed points in time and their slopes of each two time points are showed in Tables 2 and 3, respectively. This separate evaluation was to prevent collinearity. After adjustment for other confounding factors, ISS, APACHE II score, ALC (D3) and ALC (D3/D1) were significantly associated with nosocomial infection; ISS, APACHE II score, ANC (D7), ALC (D3), ANC (D7/D3) and ALC (D3/D1) were significantly associated with sepsis; ISS, APACHE II score, GCS, ALC (D3), ALC (D7), AMC (D7) and ALC (D3/D1) were significantly associated with mortality. 
Table 2

Univariate and multivariate logistic regression analysis including immune cell counts at fixed time points

\begin{tabular}{|c|c|c|c|c|c|c|c|c|c|c|c|c|}
\hline \multirow[t]{4}{*}{ Variables } & \multicolumn{4}{|c|}{ Nosocomial infection } & \multicolumn{4}{|l|}{ Sepsis } & \multicolumn{4}{|c|}{ Mortality } \\
\hline & \multicolumn{2}{|c|}{$\begin{array}{l}\text { Univariate } \\
\text { analysis }\end{array}$} & \multicolumn{2}{|c|}{$\begin{array}{l}\text { Multivariate } \\
\text { analysis }\end{array}$} & \multicolumn{2}{|c|}{$\begin{array}{l}\text { Univariate } \\
\text { analysis }\end{array}$} & \multicolumn{2}{|c|}{$\begin{array}{l}\text { Multivariate } \\
\text { analysis }\end{array}$} & \multicolumn{2}{|c|}{$\begin{array}{l}\text { Univariate } \\
\text { analysis }\end{array}$} & \multicolumn{2}{|c|}{$\begin{array}{l}\text { Multivariate } \\
\text { analysis }\end{array}$} \\
\hline & $\begin{array}{l}\text { OR } \\
(95 \%\end{array}$ & $p$ & $\begin{array}{l}\text { OR } \\
(95 \%\end{array}$ & $\mathbf{p}$ & $\begin{array}{l}\text { OR } \\
(95 \%\end{array}$ & $\mathrm{p}$ & $\begin{array}{l}\text { OR } \\
(95 \%\end{array}$ & $p$ & $\begin{array}{l}\text { OR } \\
(95 \%\end{array}$ & $\mathbf{p}$ & $\begin{array}{l}\text { OR } \\
(95 \%\end{array}$ & $\mathbf{p}$ \\
\hline & $\mathrm{Cl})$ & & $\mathrm{Cl})$ & & $\mathrm{Cl})$ & & $\mathrm{Cl})$ & & $\mathrm{Cl})$ & & $\mathrm{Cl})$ & \\
\hline Age & $\begin{array}{l}1.02 \\
(1.00 \\
1.03)\end{array}$ & 0.027 & $\begin{array}{l}1.01 \\
(0.99 \\
1.03)\end{array}$ & 0.348 & $\begin{array}{l}1.02 \\
(1.00 \\
1.03)\end{array}$ & 0.023 & $\begin{array}{l}1.00 \\
(0.98 \\
1.02)\end{array}$ & 0.768 & $\begin{array}{l}1.03 \\
(1.01 \\
1.05)^{\prime}\end{array}$ & 0.001 & $\begin{array}{l}0.99 \\
(0.97 \\
1.02)\end{array}$ & 0.580 \\
\hline ISS & $\begin{array}{l}1.10 \\
(1.06, \\
1.13)\end{array}$ & $\begin{array}{l}<.001 \\
0.00\end{array}$ & $\begin{array}{l}1.04 \\
(1.01 \\
1.09)^{\prime}\end{array}$ & 0.028 & $\begin{array}{l}1.10 \\
(1.07 \\
1.14)^{\prime}\end{array}$ & $\begin{array}{l}< \\
0.001\end{array}$ & $\begin{array}{l}1.05 \\
(1.01 \\
109)\end{array}$ & 0.015 & $\begin{array}{l}1.08 \\
(1.05 \\
1.11)\end{array}$ & $\begin{array}{l}< \\
0.001\end{array}$ & $\begin{array}{l}1.05 \\
(1.03 \\
1.08)\end{array}$ & 0.010 \\
\hline $\begin{array}{l}\text { APACHE } \\
\text { II }\end{array}$ & $\begin{array}{l}1.14 \\
(1.10 \\
1.19\end{array}$ & $\begin{array}{l}<.001 \\
0.00\end{array}$ & $\begin{array}{l}1.10 \\
(1.04 \\
1.17)\end{array}$ & 0.001 & $\begin{array}{l}1.18 \\
(1.13 \\
1.23)\end{array}$ & $\begin{array}{l}< \\
0.001\end{array}$ & $\begin{array}{l}1.18 \\
(1.10 \\
1.26)\end{array}$ & $\begin{array}{l}< \\
0.001\end{array}$ & $\begin{array}{l}1.24 \\
(1.18 \\
1.30)\end{array}$ & $\begin{array}{l}< \\
0.001\end{array}$ & $\begin{array}{l}1.28 \\
(1.18 \\
1.38)\end{array}$ & $\begin{array}{l}< \\
0.001\end{array}$ \\
\hline GCS & $\begin{array}{l}0.89 \\
(0.83 \\
0.94)\end{array}$ & $\begin{array}{l}< \\
0.001\end{array}$ & $\begin{array}{l}1.05 \\
(0.97 \\
1.13)\end{array}$ & 0.225 & $\begin{array}{l}0.84 \\
(0.79 \\
0.89)\end{array}$ & $\begin{array}{l}<.001 \\
0.00\end{array}$ & $\begin{array}{l}1.07 \\
(0.98 \\
1.16)\end{array}$ & 0.138 & $\begin{array}{l}0.82 \\
(0.77 \\
0.88)\end{array}$ & $\begin{array}{l}< \\
0.001\end{array}$ & $\begin{array}{l}1.09 \\
(1.00 \\
1.18)\end{array}$ & 0.043 \\
\hline ANC (D1) & $\begin{array}{l}1.06 \\
(0.01 \\
1.11)\end{array}$ & 0.021 & $\begin{array}{l}1.01 \\
(0.95 \\
1.07)\end{array}$ & 0.802 & $\begin{array}{l}1.05 \\
(1.01 \\
1.10)\end{array}$ & 0.029 & $\begin{array}{l}0.99 \\
(0.93 \\
1.05)\end{array}$ & 0.638 & $\begin{array}{l}1.05 \\
(1.00 \\
1.11)\end{array}$ & 0.157 & & \\
\hline ANC (D3) & $\begin{array}{l}1.14 \\
(1.08 \\
1.22)\end{array}$ & $\begin{array}{l}<.001 \\
0.00\end{array}$ & $\begin{array}{l}1.02 \\
(0.94 \\
1.11)\end{array}$ & 0.702 & $\begin{array}{l}1.16 \\
(1.09 \\
1.23)^{\prime}\end{array}$ & $\begin{array}{l}<.001 \\
0.00\end{array}$ & $\begin{array}{l}1.08 \\
(0.99 \\
1.18)\end{array}$ & 0.094 & $\begin{array}{l}1.06 \\
(1.01 \\
1.12)^{\prime}\end{array}$ & 0.034 & $\begin{array}{l}1.05 \\
(0.95 \\
1.17)\end{array}$ & 0.369 \\
\hline ANC (D7) & & & & & $\begin{array}{l}1.23 \\
(1.16 \\
1.30)\end{array}$ & $\begin{array}{l}<.001 \\
0.00\end{array}$ & $\begin{array}{l}1.17 \\
(1.08 \\
1.26)\end{array}$ & $\begin{array}{l}< \\
0.001\end{array}$ & $\begin{array}{l}1.07 \\
(1.03 \\
1.12)\end{array}$ & 0.003 & $\begin{array}{l}1.07 \\
(0.98 \\
1.16)\end{array}$ & 0.114 \\
\hline ALC (D1) & $\begin{array}{l}1.04 \\
(0.65 \\
1.67)\end{array}$ & 0.879 & & & $\begin{array}{l}0.95 \\
(0.57 \\
1.59)\end{array}$ & 0.851 & & & $\begin{array}{l}1.08 \\
(0.64 \\
1.91)\end{array}$ & 0.806 & & \\
\hline ALC (D3) & $\begin{array}{l}0.55 \\
(0.37 \\
0.83)\end{array}$ & 0.004 & $\begin{array}{l}0.48 \\
(0.29 \\
0.79)\end{array}$ & 0.003 & $\begin{array}{l}0.55 \\
(0.34 \\
0.89)\end{array}$ & 0.015 & $\begin{array}{l}0.47 \\
(0.24 \\
0.91)\end{array}$ & 0.026 & $\begin{array}{l}0.34 \\
(0.18 \\
0.65)\end{array}$ & 0.001 & $\begin{array}{l}0.38 \\
(0.16 \\
0.89)\end{array}$ & 0.025 \\
\hline ALC (D7) & & & & & $\begin{array}{l}0.54 \\
(0.35 \\
0.83)\end{array}$ & 0.005 & $\begin{array}{l}0.60 \\
(0.33 \\
1.08)\end{array}$ & 0.088 & $\begin{array}{l}0.15 \\
(0.08 \\
0.30)\end{array}$ & $<.001$ & $\begin{array}{l}0.27 \\
(0.11 \\
0.63)\end{array}$ & 0.003 \\
\hline $\begin{array}{l}\text { AMC } \\
\text { (D1) }\end{array}$ & $\begin{array}{l}1.47 \\
(0.86 \\
2.52)\end{array}$ & 0.258 & & & $\begin{array}{l}1.36 \\
(0.79 \\
2.33)\end{array}$ & 0.270 & & & $\begin{array}{l}1.38 \\
(0.75 \\
2.54)\end{array}$ & 0.308 & & \\
\hline $\begin{array}{l}\text { AMC } \\
\text { (D3) }\end{array}$ & $\begin{array}{l}1.48 \\
(0.84 \\
2.58)\end{array}$ & 0.272 & & & $\begin{array}{l}1.29 \\
(0.73 \\
2.26)\end{array}$ & 0.379 & & & $\begin{array}{l}0.47 \\
(0.22 \\
1.00)\end{array}$ & 0.049 & $\begin{array}{l}1.32 \\
(0.42 \\
4.13)\end{array}$ & 0.637 \\
\hline $\begin{array}{l}\text { AMC } \\
\text { (D7) }\end{array}$ & & & & & $\begin{array}{l}2.23 \\
(1.28 \\
3.53)\end{array}$ & 0.003 & $\begin{array}{l}1.73 \\
(0.76 \\
3.94)\end{array}$ & 0.190 & $\begin{array}{l}0.24 \\
(0.11 \\
0.52)\end{array}$ & $\begin{array}{l}< \\
0.001\end{array}$ & $\begin{array}{l}0.21 \\
(0.06 \\
0.68)\end{array}$ & 0.023 \\
\hline
\end{tabular}


Table 3

Univariate and multivariate logistic regression analyses including slopes of immune cell counts over time

\begin{tabular}{|c|c|c|c|c|c|c|c|c|c|c|c|c|}
\hline \multirow[t]{4}{*}{ Variables } & \multicolumn{4}{|c|}{ Nosocomial infection } & \multicolumn{4}{|l|}{ Sepsis } & \multicolumn{4}{|l|}{ Mortality } \\
\hline & \multicolumn{2}{|c|}{$\begin{array}{l}\text { Univariate } \\
\text { analysis }\end{array}$} & \multicolumn{2}{|c|}{$\begin{array}{l}\text { Multivariate } \\
\text { analysis }\end{array}$} & \multicolumn{2}{|c|}{$\begin{array}{l}\text { Univariate } \\
\text { analysis }\end{array}$} & \multicolumn{2}{|c|}{$\begin{array}{l}\text { Multivariate } \\
\text { analysis }\end{array}$} & \multicolumn{2}{|c|}{$\begin{array}{l}\text { Univariate } \\
\text { analysis }\end{array}$} & \multicolumn{2}{|c|}{$\begin{array}{l}\text { Multivariate } \\
\text { analysis }\end{array}$} \\
\hline & $\begin{array}{l}\text { OR } \\
(95 \%\end{array}$ & $p$ & $\begin{array}{l}\text { OR } \\
(95 \%\end{array}$ & $p$ & $\begin{array}{l}\text { OR } \\
(95 \%\end{array}$ & $\mathrm{p}$ & $\begin{array}{l}\text { OR } \\
(95 \%\end{array}$ & $\mathbf{p}$ & $\begin{array}{l}\text { OR } \\
(95 \%\end{array}$ & $\mathbf{p}$ & $\begin{array}{l}\text { OR } \\
(95 \%\end{array}$ & $p$ \\
\hline & $\mathrm{Cl})$ & & $\mathrm{Cl})$ & & $\mathrm{Cl})$ & & $\mathrm{Cl})$ & & $\mathrm{Cl})$ & & $\mathrm{Cl})$ & \\
\hline Age & $\begin{array}{l}1.02 \\
(1.00 \\
1.03)\end{array}$ & 0.027 & $\begin{array}{l}1.01 \\
(0.99 \\
1.02)\end{array}$ & 0.460 & $\begin{array}{l}1.02 \\
(1.00 \\
1.03)\end{array}$ & 0.023 & $\begin{array}{l}1.01 \\
(0.99 \\
1.03)\end{array}$ & 0.344 & $\begin{array}{l}1.03 \\
(1.01 \\
1.05)\end{array}$ & 0.001 & $\begin{array}{l}1.02 \\
(1.00 \\
1.04)\end{array}$ & 0.122 \\
\hline ISS & $\begin{array}{l}1.10 \\
(1.06, \\
1.13)\end{array}$ & $\begin{array}{l}< \\
0.001\end{array}$ & $\begin{array}{l}1.07 \\
(1.03, \\
1.11)\end{array}$ & $\begin{array}{l}< \\
0.001\end{array}$ & $\begin{array}{l}1.10 \\
(1.07 \\
1.14)\end{array}$ & $\begin{array}{l}< \\
0.001\end{array}$ & $\begin{array}{l}1.06 \\
(1.03, \\
1.10)\end{array}$ & 0.001 & $\begin{array}{l}1.08 \\
(1.05, \\
1.11)\end{array}$ & $\begin{array}{l}< \\
0.001\end{array}$ & $\begin{array}{l}1.07 \\
(1.04 \\
1.10)\end{array}$ & 0.013 \\
\hline $\begin{array}{l}\text { APACHE } \\
\text { II }\end{array}$ & $\begin{array}{l}1.14 \\
(1.10 \\
1.19\end{array}$ & $\begin{array}{l}< \\
0.001\end{array}$ & $\begin{array}{l}1.10 \\
(1.05 \\
1.17)\end{array}$ & $\begin{array}{l}< \\
0.001\end{array}$ & $\begin{array}{l}1.18 \\
(1.13 \\
1.23)\end{array}$ & $\begin{array}{l}< \\
0.001\end{array}$ & $\begin{array}{l}1.16 \\
(1.09 \\
1.24)\end{array}$ & $\begin{array}{l}< \\
0.001\end{array}$ & $\begin{array}{l}1.24 \\
(1.18, \\
1.30)\end{array}$ & $\begin{array}{l}< \\
0.001\end{array}$ & $\begin{array}{l}1.30 \\
(1.20 \\
1.41)\end{array}$ & $\begin{array}{l}< \\
0.001\end{array}$ \\
\hline GCS & $\begin{array}{l}0.89 \\
(0.83 \\
0.94)\end{array}$ & $\begin{array}{l}< \\
0.001\end{array}$ & $\begin{array}{l}1.02 \\
(0.95 \\
1.09)\end{array}$ & 0.610 & $\begin{array}{l}0.84 \\
(0.79 \\
0.89)\end{array}$ & $\begin{array}{l}<.001 \\
0.00\end{array}$ & $\begin{array}{l}1.04 \\
(0.96 \\
1.13)\end{array}$ & 0.377 & $\begin{array}{l}0.82 \\
(0.77 \\
0.88)\end{array}$ & $<.001$ & $\begin{array}{l}1.08 \\
(1.00 \\
1.18)\end{array}$ & 0.044 \\
\hline $\begin{array}{l}\text { ANC } \\
\text { (D3/D1) }\end{array}$ & $\begin{array}{l}1.48 \\
(0.99 \\
2.21)\end{array}$ & 0.158 & & & $\begin{array}{l}1.52 \\
(1.05 \\
2.19)\end{array}$ & 0.027 & $\begin{array}{l}1.49 \\
(0.94 \\
2.37)\end{array}$ & 0.089 & $\begin{array}{l}1.18 \\
(0.79 \\
1.76)\end{array}$ & 0.413 & & \\
\hline $\begin{array}{l}\text { ANC } \\
\text { (D7/D3) }\end{array}$ & & & & & $\begin{array}{l}1.71 \\
(1.24 \\
2.35)\end{array}$ & 0.001 & $\begin{array}{l}1.63 \\
(1.13 \\
2.33)\end{array}$ & 0.008 & $\begin{array}{l}1.13 \\
(0.80 \\
1.59)\end{array}$ & 0.479 & & \\
\hline $\begin{array}{l}\text { ALC } \\
\text { (D3/D1) }\end{array}$ & $\begin{array}{l}0.70 \\
(0.56 \\
0.89)\end{array}$ & 0.004 & $\begin{array}{l}0.72 \\
(0.56 \\
0.93)\end{array}$ & 0.010 & $\begin{array}{l}0.70 \\
(0.53 \\
0.94)\end{array}$ & 0.017 & $\begin{array}{l}0.71 \\
(0.55 \\
0.96)\end{array}$ & 0.046 & $\begin{array}{l}0.69 \\
(0.48 \\
0.97)\end{array}$ & 0.035 & $\begin{array}{l}0.72 \\
(0.51 \\
0.99)\end{array}$ & 0.049 \\
\hline $\begin{array}{l}\text { ALC } \\
\text { (D7/D3) }\end{array}$ & & & & & $\begin{array}{l}1.33 \\
(1.06, \\
1.66)\end{array}$ & 0.014 & $\begin{array}{l}1.16 \\
(0.86 \\
1.55)\end{array}$ & 0.338 & $\begin{array}{l}0.86 \\
(0.66 \\
1.18)\end{array}$ & 0.404 & & \\
\hline $\begin{array}{l}\text { AMC } \\
\text { (D3/D1) }\end{array}$ & $\begin{array}{l}1.03 \\
(0.93 \\
1.13)\end{array}$ & 0.616 & & & $\begin{array}{l}1.05 \\
(0.95 \\
1.17)\end{array}$ & 0.316 & & & $\begin{array}{l}1.06 \\
(0.96 \\
1.16)\end{array}$ & 0.239 & & \\
\hline $\begin{array}{l}\text { AMC } \\
\text { (D7/D3) }\end{array}$ & & & & & $\begin{array}{l}1.21 \\
(1.02, \\
1.44)\end{array}$ & 0.029 & $\begin{array}{l}1.09 \\
(0.89 \\
1.34)\end{array}$ & 0.389 & $\begin{array}{l}0.91 \\
(0.72 \\
1.16)\end{array}$ & 0.447 & & \\
\hline $\begin{array}{l}\text { Table shov } \\
\text { multivariat } \\
\text { Physiology } \\
\text { lymphocyt }\end{array}$ & $\begin{array}{l}\text { he re } \\
\text { gisti } \\
\text { d Ch } \\
\text { unt; }\end{array}$ & $\begin{array}{l}\text { sof } \\
\text { yress } \\
\mathrm{CHe} \\
\mathrm{H}=\mathrm{a}\end{array}$ & $\begin{array}{l}\text { bles } \\
\text { analy } \\
\text { Evalu } \\
\text { ate m }\end{array}$ & $\begin{array}{l}\text { ted } t \\
\text { OR } \\
\text { in II } \\
\text { cyte }\end{array}$ & $\begin{array}{l}\text { mial } \\
\text { tio; } 0 \\
\text { is = C } \\
3 / D\end{array}$ & $\begin{array}{l}\text { ection } \\
\text { confid } \\
\text { gow c } \\
\text { day 3/ }\end{array}$ & $\begin{array}{l}\text { epsis } \\
\text { e int } \\
\text { a sc } \\
1 ; D\end{array}$ & $\begin{array}{l}\text { d mor } \\
\text { l; ISS } \\
\text { ANC = } \\
3=\text { da }\end{array}$ & $\begin{array}{l}\text { etermine } \\
\text { Severity } \\
\text { te neutro } \\
3 \text {. }\end{array}$ & $\begin{array}{l}\text { oy uni } \\
\text { ore; } A \\
\text { il cou }\end{array}$ & $\begin{array}{l}\text { riate an } \\
\mathrm{ACHE} I \mathrm{I} \\
\mathrm{ALC}=\end{array}$ & $\begin{array}{l}\text { cute } \\
\text { solute }\end{array}$ \\
\hline
\end{tabular}

Predictive value

The neutrophil-lymphocyte ratio (NLR) has been widely proved to be a simple and reliable marker for assessing systemic inflammation and prognosis in non-traumatic and traumatic patients [1, 18-23]. In our study, we validated the predicted value of NLR (D3) and NLR (D7) using ROC curve given their differences between each two groups (Fig. 5). Since the median time of nosocomial infection was day 6 (data not shown) in our study, which may be the cause of the rise of ANC (D7), we did not analyze the effect of NLR (D7) on nosocomial infection. The results show that NLR (D7) has a larger area under the ROC curve (AUC) in predicting the risk of sepsis and mortality than NLR (D3) (Table 4). Then, we selected NLR which has a larger AUC and the independent risk factors of nosocomial infection, sepsis and mortality (Tables 2 and 3) for comparison. As depicted in Fig. 6A, 6B, 
$6 \mathrm{C}$ and Table 5, APACHE II score has the best predicted value for the risk of nosocomial infection, sepsis and mortality (AUC: 0.71 , 0.80 and 0.87 , respectively). ISS for nosocomial infection and NLR (D7) for sepsis and mortality were the next best (AUC: $0.69,0.76$ and 0.77 , respectively).

Table 4

Predictive value of neutrophil-lymphocyte ratio for nosocomial infection, sepsis and mortality

\begin{tabular}{|c|c|c|c|c|c|c|c|}
\hline & AUC & $\begin{array}{l}\text { Cut- } \\
\text { off }\end{array}$ & $\begin{array}{l}\text { Sensitivity (95\% } \\
\mathrm{Cl)}\end{array}$ & $\begin{array}{l}\text { Specificity (95\% } \\
\mathrm{Cl})\end{array}$ & PPV (95\% Cl) & NPV (95\% Cl) & $p$ \\
\hline \multicolumn{8}{|c|}{$\begin{array}{l}\text { Nosocomial } \\
\text { infection }\end{array}$} \\
\hline NLR (D3) & $\begin{array}{l}0.66(0.61, \\
0.71)\end{array}$ & $\overrightarrow{6} .74$ & $\begin{array}{l}72.44(66.1, \\
78.2)\end{array}$ & $\begin{array}{l}52.98(44.7 \\
61.1)\end{array}$ & $\begin{array}{l}69.7(65.6, \\
73.5)\end{array}$ & $\begin{array}{l}56.3(49.9, \\
62.6)\end{array}$ & $\begin{array}{l}<.001 \\
0.00\end{array}$ \\
\hline \multicolumn{8}{|l|}{ Sepsis } \\
\hline NLR (D3) & $\begin{array}{l}0.72(0.67 \\
0.76)\end{array}$ & 8.57 & $\begin{array}{l}78.45(69.9, \\
85.5)\end{array}$ & $\begin{array}{l}59.92(53.7 \\
65.9)\end{array}$ & $\begin{array}{l}46.4(42.1 \\
50.8)\end{array}$ & $\begin{array}{l}86.3(81.4, \\
90.0)\end{array}$ & $\begin{array}{l}<.001 \\
0.0\end{array}$ \\
\hline NLR (D7) & $\begin{array}{l}0.76(071, \\
0.80)\end{array}$ & 7.34 & $\begin{array}{l}81.03(72.7, \\
87.7)\end{array}$ & $\begin{array}{l}61.30(55.1 \\
67.2)\end{array}$ & $\begin{array}{l}48.2(43.8 \\
52.6)\end{array}$ & $\begin{array}{l}87.9(83.1, \\
91.5)\end{array}$ & $\begin{array}{l}<.001 \\
0.00\end{array}$ \\
\hline \multicolumn{8}{|l|}{ Mortality } \\
\hline NLR (D3) & $\begin{array}{l}0.69(0.64, \\
0.73)\end{array}$ & 8.01 & $\begin{array}{l}83.33 \text { (72.7, } \\
91.1)\end{array}$ & $\begin{array}{l}50.98(45.2 \\
56.7)\end{array}$ & $\begin{array}{l}28.6(25.5, \\
31.8)\end{array}$ & $\begin{array}{l}92.9(88.5, \\
95.7)\end{array}$ & $\begin{array}{l}<.001 \\
0.00\end{array}$ \\
\hline NLR (D7) & $\begin{array}{l}0.77(0.72, \\
0.81)\end{array}$ & $\overrightarrow{14.23}$ & $\begin{array}{l}56.94(44.7 \\
68.6)\end{array}$ & $\begin{array}{l}86.23(81.8 \\
89.9)\end{array}$ & $\begin{array}{l}49.4(40.9 \\
58.0)\end{array}$ & $\begin{array}{l}89.5(86.6, \\
91.7)\end{array}$ & $\begin{array}{l}< \\
0.001\end{array}$ \\
\hline
\end{tabular}


Table 5

Predictive value of various indexes for nosocomial infection, sepsis and mortality

\begin{tabular}{|c|c|c|c|c|c|c|c|}
\hline & AUC & $\begin{array}{l}\text { Cut- } \\
\text { off }\end{array}$ & $\begin{array}{l}\text { Sensitivity (95\% } \\
\mathrm{Cl})\end{array}$ & $\begin{array}{l}\text { Specificity (95\% } \\
\mathrm{Cl})\end{array}$ & PPV (95\% Cl) & NPV $(95 \% \mathrm{Cl})$ & p \\
\hline \multicolumn{8}{|l|}{$\begin{array}{l}\text { Nosocomial } \\
\text { infection }\end{array}$} \\
\hline ISS & $\begin{array}{l}0.69(0.65 \\
0.73)\end{array}$ & $>22$ & $\begin{array}{l}67.65(61.3 \\
73.5)\end{array}$ & $\begin{array}{l}63.00(55.9 \\
69.7)\end{array}$ & $\begin{array}{l}68.5(64.0, \\
72.7)\end{array}$ & $\begin{array}{l}62.1(57.0, \\
66.9)\end{array}$ & $\begin{array}{l}< \\
0.001\end{array}$ \\
\hline APACHE II & $\begin{array}{l}0.71(0.67, \\
0.75)\end{array}$ & $>11$ & $\begin{array}{l}61.76 \\
(55.3,68.0)\end{array}$ & $\begin{array}{l}73.00 \\
(66.3,79.0)\end{array}$ & $\begin{array}{l}73.1 \\
(68.0,77.7)\end{array}$ & $\begin{array}{l}61.6 \\
(57.2,65.8)\end{array}$ & $\dot{0} 001$ \\
\hline ALC (D3) & $\begin{array}{l}0.62(0.57, \\
0.68)\end{array}$ & $\begin{array}{l}\leq \\
0.83\end{array}$ & $\begin{array}{l}67.76(59.7 \\
75.1)\end{array}$ & $\begin{array}{l}53.54(46.8 \\
60.2)\end{array}$ & $\begin{array}{l}49.5(45.1 \\
54.0)\end{array}$ & $\begin{array}{l}71.2(65.6, \\
76.2)\end{array}$ & $\begin{array}{l}< \\
0.001\end{array}$ \\
\hline ALC (D3/D1) & $\begin{array}{l}0.68(0.64, \\
0.73)\end{array}$ & $\leq$ & $\begin{array}{l}79.65 \\
(73.8,84.7)\end{array}$ & $\begin{array}{l}46.05 \\
(37.9,54.3)\end{array}$ & $\begin{array}{l}68.7 \\
(65.1,72.1)\end{array}$ & $\begin{array}{l}60.3 \\
(52.7,67.5)\end{array}$ & $\begin{array}{l}< \\
0.001\end{array}$ \\
\hline NLR (D3) & $\begin{array}{l}0.66(0.61, \\
0.71)\end{array}$ & 3.74 & $\begin{array}{l}72.44(66.1 \\
78.2)\end{array}$ & $\begin{array}{l}52.98(44.7 \\
61.1)\end{array}$ & $\begin{array}{l}69.7(65.6, \\
73.5)\end{array}$ & $\begin{array}{l}56.3(49.9, \\
62.6)\end{array}$ & $\dot{0.001}$ \\
\hline \multicolumn{8}{|l|}{ Sepsis } \\
\hline ISS & $\begin{array}{l}0.71(0.67 \\
0.75)\end{array}$ & $>27$ & $\begin{array}{l}55.46(46.1 \\
64.60)\end{array}$ & $\begin{array}{l}77.12(72.1 \\
81.6)\end{array}$ & $\begin{array}{l}47.5(41.1, \\
53.9)\end{array}$ & $\begin{array}{l}82.3(79.0, \\
85.10\end{array}$ & $\begin{array}{l}< \\
0.001\end{array}$ \\
\hline APACHE II & $\begin{array}{l}0.80(0.76 \\
0.84)\end{array}$ & $>11$ & $\begin{array}{l}81.90(73.7 \\
88.4)\end{array}$ & $\begin{array}{l}65.31(59.8 \\
70.5)\end{array}$ & $\begin{array}{l}46.1(41.9 \\
50.4)\end{array}$ & $\begin{array}{l}90.9(87.0, \\
93.7)\end{array}$ & $\begin{array}{l}< \\
0.001\end{array}$ \\
\hline ANC (D7) & $\begin{array}{l}0.75(0.70 \\
0.79)\end{array}$ & $\begin{array}{l}> \\
11.27\end{array}$ & $\begin{array}{l}58.62(49.1 \\
67.7)\end{array}$ & $\begin{array}{l}83.59 \\
87.9)\end{array}$ & $\begin{array}{l}61.3(53.6, \\
68.4)\end{array}$ & $\begin{array}{l}82.0(78.5, \\
85.1)\end{array}$ & $\begin{array}{l}<.001 \\
0.001\end{array}$ \\
\hline ALC (D3) & $\begin{array}{l}0.64(0.59 \\
0.69)\end{array}$ & $\leq$ & $\begin{array}{l}80.17 \text { (71.7, } \\
87.0)\end{array}$ & $\begin{array}{l}46.18(40.0 \\
52.4\end{array}$ & $\begin{array}{l}39.7(36.3 \\
43.2)\end{array}$ & $\begin{array}{l}84.0(78.1, \\
88.6)\end{array}$ & $\begin{array}{l}<.001 \\
0.001\end{array}$ \\
\hline ANC (D7/D3) & $\begin{array}{l}0.71(0.57, \\
0.67)\end{array}$ & $\overrightarrow{1.13}$ & $\begin{array}{l}92.24 \\
(85.8,96.4)\end{array}$ & $\begin{array}{l}44.66 \\
(38.5,50.9)\end{array}$ & $\begin{array}{l}42.5 \\
(39.5,45.4)\end{array}$ & $\begin{array}{l}92.9 \\
(87.2,96.1)\end{array}$ & $\begin{array}{l}< \\
0.001\end{array}$ \\
\hline ALC (D3/D1) & $\begin{array}{l}0.75(0.70 \\
0.79)\end{array}$ & $\begin{array}{l}\leq \\
0.97\end{array}$ & $\begin{array}{l}74.14 \\
(65.2,81.8)\end{array}$ & $\begin{array}{l}64.5 \\
(58.4,70.3)\end{array}$ & $\begin{array}{l}48.0 \\
(43.2,52.9)\end{array}$ & $\begin{array}{l}84.9 \\
(80.3,88.6)\end{array}$ & $\begin{array}{l}< \\
0.001\end{array}$ \\
\hline NLR (D7) & $\begin{array}{l}0.76(071 \\
0.80)\end{array}$ & 7.34 & $\begin{array}{l}81.03(72.7 \\
87.7)\end{array}$ & $\begin{array}{l}61.30(55.1, \\
67.2)\end{array}$ & $\begin{array}{l}48.2(43.8 \\
52.6)\end{array}$ & $\begin{array}{l}87.9(83.1, \\
91.5)\end{array}$ & $\dot{0} 001$ \\
\hline \multicolumn{8}{|l|}{ Mortality } \\
\hline ISS & $\begin{array}{l}0.70(0.63, \\
0.76)\end{array}$ & $>23$ & $\begin{array}{l}80.77(70.3 \\
88.8)\end{array}$ & $\begin{array}{l}52.50(47.2, \\
57.8)\end{array}$ & $\begin{array}{l}26.9(24.0 \\
30.0)\end{array}$ & $\begin{array}{l}92.6(88.8, \\
95.3)\end{array}$ & $\begin{array}{l}< \\
0.001\end{array}$ \\
\hline APACHE II & $\begin{array}{l}0.87(0.83, \\
0.91)\end{array}$ & $>14$ & $\begin{array}{l}80.52(69.9 \\
88.7)\end{array}$ & $\begin{array}{l}83.39 \text { (78.7, } \\
87.4)\end{array}$ & $\begin{array}{l}55.4(48.5 \\
62.0)\end{array}$ & $\begin{array}{l}94.4(91.4, \\
96.4)\end{array}$ & $\dot{0} 001$ \\
\hline GCS & $\begin{array}{l}0.70(0.65 \\
0.75)\end{array}$ & $\leq 13$ & $\begin{array}{l}67.57 \\
(55.7,78.0)\end{array}$ & $\begin{array}{l}63.7 \\
(58.0,69.1)\end{array}$ & $\begin{array}{l}31.3 \\
(26.8,36.1)\end{array}$ & $\begin{array}{l}88.9 \\
(85.1,91.9)\end{array}$ & $\begin{array}{l}<.001 \\
0.001\end{array}$ \\
\hline ALC (D3) & $\begin{array}{l}0.68(0.63, \\
0.75)\end{array}$ & $\leq$ & $\begin{array}{l}70.83(58.9 \\
81.0)\end{array}$ & $\begin{array}{l}61.11(55.4, \\
66.6)\end{array}$ & $\begin{array}{l}30.0(25.9, \\
34.5)\end{array}$ & $\begin{array}{l}89.9(86.0, \\
92.8)\end{array}$ & $\dot{0.001}$ \\
\hline ALC (D7) & $\begin{array}{l}0.75(0.71 \\
0.80)\end{array}$ & $\leq$ & $\begin{array}{l}75.00(63.4 \\
84.5)\end{array}$ & $\begin{array}{l}70.59(65.1 \\
75.6)\end{array}$ & $\begin{array}{l}37.5(32.5 \\
42.8)\end{array}$ & $\begin{array}{l}92.3(88.9, \\
94.7)\end{array}$ & $\begin{array}{l}< \\
0.001\end{array}$ \\
\hline AMC (D7) & $\begin{array}{l}0.65(0.60, \\
0.70)\end{array}$ & $\leq$ & $\begin{array}{l}43.06(31.4, \\
55.3)\end{array}$ & $\begin{array}{l}81.70(76.9 \\
85.9)\end{array}$ & $\begin{array}{l}35.6(27.9 \\
44.1)\end{array}$ & $\begin{array}{l}85.9(83.2, \\
88.2)\end{array}$ & $\dot{0} 001$ \\
\hline
\end{tabular}

$\mathrm{AUC}=$ area under the ROC; $\mathrm{Cl}$ = confidence interval; $\mathrm{PPV}=$ positive predictive value; NPV = negative predictive value; ISS = Injury Severity Score; APACHE II = Acute Physiology and Chronic Health Evaluation II score; GCS = Glasgow coma scale; ANC = absolute neutrophil count; $A L C=$ absolute lymphocyte count; $A M C$ = absolute monocyte count; NLR = neutrophil-lymphocyte ratio; D3 = day $3 ; \mathrm{D} 7=$ day $7 ; \mathrm{D} 3 / \mathrm{D} 1$ = day $3 /$ day $1 ; \mathrm{D} 7 / \mathrm{D} 3$ = day $7 /$ day 3 . 


\begin{tabular}{|c|c|c|c|c|c|c|c|}
\hline & AUC & $\begin{array}{l}\text { Cut- } \\
\text { off }\end{array}$ & $\begin{array}{l}\text { Sensitivity (95\% } \\
\mathrm{Cl})\end{array}$ & $\begin{array}{l}\text { Specificity (95\% } \\
\mathrm{Cl})\end{array}$ & PPV (95\% Cl) & NPV (95\% Cl) & $p$ \\
\hline ALC (D3/D1) & $\begin{array}{l}0.76(0.71 \\
0.80)\end{array}$ & $\leq .91$ & $\begin{array}{l}79.17 \\
(68.0,87.8)\end{array}$ & $\begin{array}{l}61.11 \\
(55.4,66.6)\end{array}$ & $\begin{array}{l}32.4 \\
(28.5,36.5)\end{array}$ & $\begin{array}{l}92.6 \\
(88.7,95.2)\end{array}$ & $\begin{array}{l}<.001 \\
0.00\end{array}$ \\
\hline NLR (D7) & $\begin{array}{l}0.77(0.72 \\
0.81)\end{array}$ & $>14.23$ & $\begin{array}{l}56.94(44.7 \\
68.6)\end{array}$ & $\begin{array}{l}86.23(81.8 \\
89.9)\end{array}$ & $\begin{array}{l}49.4(40.9 \\
58.0)\end{array}$ & $\begin{array}{l}89.5(86.6, \\
91.7)\end{array}$ & $\begin{array}{l}< \\
0.001\end{array}$ \\
\hline \multicolumn{8}{|c|}{$\begin{array}{l}\text { AUC = area under the } \mathrm{ROC} ; \mathrm{Cl}=\text { confidence interval; } \mathrm{PPV}=\text { positive predictive value; } \mathrm{NPV}=\text { negative predictive value; ISS = Injury } \\
\text { Severity Score; } \mathrm{APACHE} \mathrm{II=Acute} \mathrm{Physiology} \mathrm{and} \mathrm{Chronic} \mathrm{Health} \mathrm{Evaluation} \mathrm{II} \mathrm{score;} \mathrm{GCS} \mathrm{=} \mathrm{Glasgow} \mathrm{coma} \mathrm{scale;} \mathrm{ANC}= \\
\text { absolute neutrophil count; } \mathrm{ALC}=\text { absolute lymphocyte count; } \mathrm{AMC}=\text { absolute monocyte count; } \mathrm{NLR}=\text { neutrophil-lymphocyte } \\
\text { ratio; } \mathrm{D} 3=\text { day } 3 ; \mathrm{D} 7=\text { day } 7 ; \mathrm{D} 3 / \mathrm{D} 1=\text { day } 3 / \text { day } 1 ; \mathrm{D} 7 / \mathrm{D} 3=\text { day } 7 / \text { day } 3 .\end{array}$} \\
\hline
\end{tabular}

Considering that the independent assessment of immune cell counts at fixed time points or their slopes of two time points is onesided, the combination of them was assessed using ROC curve. The results are shown in Fig. 6 and Table 6. For nosocomial infection, the combination of ALC (D3/D1) and ALC (D3) had better predicted value than APACHE II score (AUC: 0.73 VS 0.71). For sepsis, the predictive value of the combination of ALC (D3/D1) and ALC (D3) was slightly better than combination of ANC (D7/D3) and ANC (D7) (AUC: 0.81 VS 0.80), and also slightly better than APACHE II score (AUC: 0.81 VS 0.80). For mortality, the combination of ALC (D3/D1) and ALC (D3) provided a better predicted value than the other two combinations (AUC: 0.84 VS 0.65 and 0.60 ). Although its predicted value was lower than APACHE II score (AUC: 0.84 VS 0.87), it was higher than all other predictors.

Table 6

Combination of immune cell counts and their slope for predicting nosocomial infection, sepsis and mortality

\begin{tabular}{|c|c|c|c|c|c|c|c|}
\hline & $\begin{array}{l}\text { AUC }(95 \% \\
\text { Cl) }\end{array}$ & $\begin{array}{l}\text { Cut- } \\
\text { off }\end{array}$ & $\begin{array}{l}\text { Sensitivity } \\
(95 \% \mathrm{Cl})\end{array}$ & $\begin{array}{l}\text { Specificity } \\
(95 \% \mathrm{Cl})\end{array}$ & $\begin{array}{l}\text { PPV }(95 \% \\
\text { Cl) }\end{array}$ & $\begin{array}{l}\text { NPV }(95 \% \\
\mathrm{Cl})\end{array}$ & $p$ \\
\hline \multicolumn{8}{|l|}{$\begin{array}{l}\text { Nosocomial } \\
\text { infection }\end{array}$} \\
\hline $\begin{array}{l}\operatorname{ALC}(D 3 / D 1)+A L C \\
\text { (D3) }\end{array}$ & $\begin{array}{l}0.73(0.69 \\
0.78)\end{array}$ & $\begin{array}{l}> \\
0.70\end{array}$ & $\begin{array}{l}66.37 \\
(59.8,72.5)\end{array}$ & $\begin{array}{l}68.42 \\
(60.4,75.7)\end{array}$ & $\begin{array}{l}75.8 \\
(70.8,80.1)\end{array}$ & $\begin{array}{l}57.8 \\
(52.5,62.9)\end{array}$ & <. \\
\hline \multicolumn{8}{|l|}{ Sepsis } \\
\hline $\begin{array}{l}\text { ANC (D7/D3) + ANC } \\
\text { (D7) }\end{array}$ & $\begin{array}{l}0.80(0.76 \\
0.85)\end{array}$ & $\begin{array}{l}> \\
0.70\end{array}$ & $\begin{array}{l}78.24 \\
(72.8,83.1)\end{array}$ & $\begin{array}{l}71.55 \\
(62.4,79.5)\end{array}$ & $\begin{array}{l}86.1 \\
(82.2,89.3)\end{array}$ & $\begin{array}{l}59.3 \\
(53.0,65.3)\end{array}$ & $<.001$ \\
\hline $\begin{array}{l}\mathrm{ALC}(\mathrm{D} 3 / \mathrm{D} 1)+\mathrm{ALC} \\
\text { (D3) }\end{array}$ & $\begin{array}{l}0.81(0.75 \\
0.84)\end{array}$ & $\begin{array}{l}> \\
0.66\end{array}$ & $\begin{array}{l}72.9 \\
(67.1,78.2)\end{array}$ & $\begin{array}{l}74.14 \\
(65.2,81.8)\end{array}$ & $\begin{array}{l}86.4 \\
(82.3,89.7)\end{array}$ & $\begin{array}{l}54.8 \\
(49.1,60.3)\end{array}$ & $\begin{array}{l}< \\
0.001\end{array}$ \\
\hline \multicolumn{8}{|l|}{ Mortality } \\
\hline $\begin{array}{l}\mathrm{ALC}(\mathrm{D} 3 / \mathrm{D} 1)+\mathrm{ALC} \\
\text { (D3) }\end{array}$ & $\begin{array}{l}0.84(0.75 \\
0.84)\end{array}$ & $\overrightarrow{0.24}$ & $\begin{array}{l}76.39 \\
(64.9,85.6)\end{array}$ & $\begin{array}{l}78.76(73.7 \\
83.2)\end{array}$ & $\begin{array}{l}45.8 \\
(39.7,52.1)\end{array}$ & $\begin{array}{l}93.4 \\
(90.3,95.6)\end{array}$ & $\begin{array}{l}<.001 \\
0.001\end{array}$ \\
\hline $\begin{array}{l}\text { AMC (D3/D1) + } \\
\text { AMC (D3) }\end{array}$ & $\begin{array}{l}0.60(0.54 \\
0.65)\end{array}$ & $\begin{array}{l}> \\
0.23\end{array}$ & $\begin{array}{l}29.17 \\
(19.0,41.1)\end{array}$ & $\begin{array}{l}90.2 \\
(86.3,93.3)\end{array}$ & $\begin{array}{l}41.2 \\
(29.9,53.5)\end{array}$ & $\begin{array}{l}84.4 \\
(82.3,86.3)\end{array}$ & 0.022 \\
\hline $\begin{array}{l}\text { AMC (D7/D3) + } \\
\text { AMC (D7) }\end{array}$ & $\begin{array}{l}0.65(0.60 \\
0.70)\end{array}$ & $\overrightarrow{0.24}$ & $\begin{array}{l}47.89 \\
(35.9,60.1)\end{array}$ & $\begin{array}{l}79.08 \\
(74.1,83.5)\end{array}$ & $\begin{array}{l}34.7 \\
(27.7,42.4)\end{array}$ & $\begin{array}{l}86.7(83.9 \\
89.2)\end{array}$ & $\begin{array}{l}< \\
0.001\end{array}$ \\
\hline \multicolumn{8}{|c|}{$\begin{array}{l}\text { Table shows the predictive values of the combination of immune cell counts at time point and their slope of each two time } \\
\text { points for predicting the risk of nosocomial infection, sepsis and mortality. AUC = area under the ROC; } \mathrm{Cl}=\text { confidence interval; } \\
\text { PPV = positive predictive value; } \mathrm{NPV}=\text { negative predictive value; } \mathrm{ANC}=\text { absolute neutrophil count; } \mathrm{ALC}=\text { absolute lymphocyte } \\
\text { count; } \mathrm{AMC}=\text { absolute monocyte count; } \mathrm{D} 3=\text { day } 3 ; \mathrm{D} 7=\text { day } 7 ; \mathrm{D} 3 / \mathrm{D} 1=\text { day } 3 / \text { day } 1 ; \mathrm{D} 7 / \mathrm{D} 3=\text { day } 7 / \text { day } 3 \text {. }\end{array}$} \\
\hline
\end{tabular}

\section{Discussion}

In this study, we comprehensively investigated the alterations of circulating ANC, ALC and AMC within 7 days in severe trauma patients and assessed the significance of these alterations. To our knowledge, this is the first time to analyze the relationship between the combination (immunocyte counts at time point and their changing trend with time) and prognosis in severe trauma 
patients. Based on the ISS, and whether nosocomial infection, sepsis or death occurred, patients were divided into $16 \leq$ ISS $\leq 24$ and $25 \leq$ ISS $\leq 74$ groups, non-nosocomial infection and nosocomial infection groups, non-sepsis and sepsis groups, and alive and death groups, respectively. Then, comparative studies were conducted between each two groups. Collectively, our data suggest that more severe damage can lead to more severe immune system interference, and the worsening alterations in circulating immune cell counts within 7 days are associated with the later occurrence of nosocomial infections, sepsis and death. In addition, the combination of immunocyte counts at time point and their slope over time could provide good predictive value for the risk of nosocomial infection, sepsis and mortality.

Neutrophils are on the front line of the body's defense against pathogenic microorganisms. They have a strong phagocytosis of pathogens. However, they can also cause damage to blood vessels and surrounding tissues by releasing lysosomal enzymes. And uncontrolled innate immune system response may make sepsis and organ failure more likely to occur [1, 21, 24]. In our study, we found that patients with more severe trauma had higher ANC within 7 days. Patients with subsequent nosocomial infection and sepsis also had higher ANC within 7 days. According to the results of slope, ANC of patients with subsequent nosocomial infection and sepsis decreased less on the 3rd day, and rebounded significantly on the 7th day. Although the rise in ANC on day 7 may be attributed to the appearance of nosocomial infections on day 6 , these results are sufficient to show that more severe trauma can lead to more severe deterioration of the innate immune system in addition to more severe damage to the body's organs. This in turn may exacerbate organ damage. The results of multivariate analysis showed that ANC (D7) and ANC (D7/D3) were independent risk factors for sepsis, however, this may be only the manifestation of nosocomial infection. Therefore, it cannot be concluded that higher ANC will increase the susceptibility to nosocomial infection and sepsis. Moreover, ANC (D7) of the death group was higher than the alive group, but multivariate analysis showed that it was not an independent risk factor for death. Nevertheless, it should be noted that the elevation of ANC is only one aspect of neutrophil abnormalities (e.g. neutrophil function is also impaired) [4, 2527]. The overall abnormal response of neutrophils may still lead to immunosuppression, which increases the risk of adverse complications and poor prognosis [5].

Lymphocytes play a central role in the anti-infective immune response due to their ability to interact with cells of the innate immune system and other cells of adaptive immunity. They are not only passive bystanders, but also play a key role in the proper regulation of the inflammatory response $[28,29]$. In contrast to the increase of ANC after trauma, ALC decreased, which has been widely reported $[8,30,31]$. Post-traumatic lymphopenia was also common in our study. More severe trauma did not initially lead to more severe lymphocyte depletion, but resulted in more slowly recovery of ALC. So that patients with higher ISS on days 3 and 7 had lower ALC. This changing trend of ALC was also applicable to patients with subsequent nosocomial infection and sepsis. Moreover, for patients with subsequent nosocomial infections and sepsis, ALC showed a tendency to recover on the 7th day, but for patients with subsequent death, ALC had no tendency to recover at all within 7 days. According to the results of slope, ALC did not recover or even decline on the 3rd day in patients with subsequent nosocomial infection, sepsis or death. In addition, multivariate analysis showed that ALC (D3) and ALC (D3/D1) were independent risk factors for nosocomial infection, sepsis and mortality, and ALC (D7) was also an independent risk factor for mortality. These results are similar to previous findings in critically ill patients such as trauma and sepsis, the extent and delayed recovery of lymphopenia have been widely reported to be related to nosocomial infection, sepsis and death [28, 30,32-35]. Taking together, the importance of immediate recovery of ALC for prognosis is obvious.

Monocytes are the precursors of macrophages, which can phagocytize pathogens, mediate and promote inflammatory responses, process and present antigens, and participate in the regulation of adaptive immune responses. Monocyte dysfunctions, such as reduced HLA-DR expression and decreased ability to release inflammatory factors, have been widely investigated in critical diseases such as severe trauma and sepsis [4, 34, 36-41]. However, there are few reports of changes in their counts. In our study, the changes in AMC were not as extensive as those in ANC and ALC. They did not differ between the lower and higher ISS groups. In patients with subsequent nosocomial infection and sepsis, they rose on day 7 and were higher than those of non-nosocomial infection and non-sepsis patients. The overall rise of AMC after trauma may be due to endogenous stimulation (damaged tissue), but this scaling new heights on day 7 may be caused by new exogenous stimulation (microbial infection). That is to say, this scaling new heights may be just a sign of nosocomial infection on day 6 . Interestingly, when comparing the alive group with the death group, we found that AMC was higher in survivors than those in non-survivors on days 3 and 7 . This result is similar to the findings of two recent studies: survivors have significantly higher AMC than non-survivors in critically ill patients such as injured and septic shock patients $[42,43]$. Furthermore, according to the results of slope, the change trend of AMC in the alive and death groups within 7 days is opposite, rising in the alive group and falling in the death group. In addition, multivariate analysis showed

Page 12/22 
that AMC (D7) is an independent risk factor for mortality. Considering the important role of monocytes and the fact that some of the existing monocytes may have functional impairment, the advantage of ensuring total number may mitigate the adverse outcome caused by dysfunction.

When analyzing the predictive value of various risk factors for nosocomial infection, sepsis and death, we innovatively combined immune cell counts at fixed time point with their slope over time. The results show that the combination provides better predictive value than alone. The combination of ALC (D3/D1) and ALC (D3) was the best predictor of nosocomial infection and sepsis, and APACHE II score was the best predictor of mortality. It is worth noting that although the predictive value of the combination of ALC (D3/D1) and ALC (D3) for mortality is not as good as APACHE II score, their predictive value is also quite high (AUC: 0.84). Therefore, the combination of ALC (D3/D1) and ALC (D3) can be regarded as an important predictive tool for post-traumatic adverse complications or poor prognosis.

This study has several limitations. Firstly, this is a single-center retrospective study. The nature of this study may lead to unintentional bias in patient selection. Besides, blood transfusion can affect the function of immune system [44-46], but the interference of blood transfusion is not excluded in our study. Finally, although we excluded patients who underwent surgery from after blood collection on day 1 to before blood collection on day 7, emergency surgery performed at admission and surgery performed 7 days after admission may also have an impact on the results. For example, emergency surgery on admission may cause additional interference to the immune system, and it is also uncertain whether the surgery 7 days after admission is related to nosocomial infection, sepsis and death. These limitations indicate that our conclusions need to be verified in the future by large prospective multi-center research with rigorous design.

\section{Conclusions}

First, more severe injuries result in more pronounced ANC elevations and more slowly ALC recovery within 7 days. The secondary injury caused by high ANC and immunosuppression caused by low ALC may be related to subsequent adverse events. Second, ALC (D3) and ALC (D3/D1) are independent risk factors for nosocomial infection and sepsis, and ALC (D3), ALC (D3/D1), ALC (D7) and AMC (D7) are independent risk factors for death. Third, the combination of ALC (D3/D1) and ALC (D3) may be used as a valuable predictor of nosocomial infection, sepsis, and mortality in the later post-traumatic stage. Last and outlook, immunization interventions such as clearing excessive dysfunctional ANC, recovering ALC as soon as possible and supplementing AMC with normal function may help to improve the prognosis.

\section{Abbreviations}

ANC: absolute neutrophil count; ALC: absolute lymphocyte count; AMC: absolute monocyte count; D1: day 1; D3: day 3; D7: day 7; D3/D1: day 3/day 1; D7/D3: day 7/day 3; ROC curve: Receiver operating characteristic curve; AUC: area under the ROC curve; MODS: multiple organ dysfunction syndrome; DAMP: damage-related molecular models; SIRS: systemic inflammatory response syndrome; CARS: compensatory anti-inflammatory response syndrome; APACHE II score: Acute Physiology and Chronic Health Evaluation II score; ISS: Injury Severity Score; GCS: Glasgow coma scale; COPD: chronic obstructive pulmonary diseases; IQR: interquartile range; $\mathrm{OR}$ : odds ratio; $\mathrm{Cl}$ : confidence interval; $\mathrm{PPV}=$ positive predictive value; NPV = negative predictive value.

\section{Declarations}

Ethics approval and consent to participate: Approval was obtained from the Medical Ethics Committee of the hospital with a waiver of informed consent.

Consent for publication: Not applicable.

Availability of data and materials: The datasets used and/or analyzed during the present study are available from the corresponding author on reasonable request.

Competing interests: All authors declare that they have no competing interests.

Funding: National Nature Science Foundation of China (No. 81571891, 81801072 and 81772129).

Page $13 / 22$ 
Authors' contributions: XJD and CTW were responsible for data acquisition, statistical assistance and data analysis. XHL were responsible for manuscript drafting and revision. XJD and ZFL were responsible for concept and design, data analysis, manuscript drafting and revision. XJD, CTW XHL, XJB and ZFL discussed the results. All authors read and approved the final manuscript.

Acknowledgements: Not applicable.

\section{References}

1.

Dilektasli E, Inaba K, Haltmeier T, Wong MD, Clark D, Benjamin ER, Lam L, Demetriades D. The prognostic value of neutrophil-tolymphocyte ratio on mortality in critically ill trauma patients. J Trauma Acute Care Surg. 2016;81(5):882-8.

2.

de Munter L, Polinder S, Lansink KW, Cnossen MC, Steyerberg EW, de Jongh MA. Mortality prediction models in the general trauma population: A systematic review. Injury. 2017;48(2):221-9.

3.

Grzalja N, Cicvaric T, Knezevic D, Kuharic J, Sustic A, Bakota B, Komen S, Tokmadzic VS. Frequency and perforin expression of different lymphocyte subpopulations in patients with lower limb fracture and thoracic injury. Injury. 2017;48(Suppl 5):1-7.

4.

Hazeldine J, Naumann DN, Toman E, Davies D, Bishop JRB, Su Z, Hampson P, Dinsdale RJ, Crombie N, Duggal NA, et al. Prehospital immune responses and development of multiple organ dysfunction syndrome following traumatic injury: A prospective cohort study. PLoS Med. 2017;14(7):e1002338.

5 .

Lord JM, Midwinter MJ, Chen YF, Belli A, Brohi K, Kovacs EJ, Koenderman L, Kubes P, Lilford RJ. The systemic immune response to trauma: an overview of pathophysiology and treatment. Lancet. 2014;384(9952):1455-65.

6.

Manson J, Cole E, De'Ath HD, Vulliamy P, Meier U, Pennington D, Brohi K. Early changes within the lymphocyte population are associated with the development of multiple organ dysfunction syndrome in trauma patients. Crit Care 2016, 20.

7.

Jo YG, Choi HJ, Kim JC, Cho YN, Kang JH, Jin HM, Kee SJ, Park YW. Deficiencies of Circulating Mucosal-associated Invariant T Cells and Natural Killer T Cells in Patients with Multiple Trauma. J Korean Med Sci. 2017;32(5):750-6.

8.

Serve R, Sturm R, Schimunek L, Stormann P, Heftrig D, Teuben MPJ, Oppermann E, Horst K, Pfeifer R, Simon TP, et al. Comparative Analysis of the Regulatory T Cells Dynamics in Peripheral Blood in Human and Porcine Polytrauma. Front Immunol. 2018;9:435.

9.

Xiao W, Mindrinos MN, Seok J, Cuschieri J, Cuenca AG, Gao H, Hayden DL, Hennessy L, Moore EE, Minei JP, et al. A genomic storm in critically injured humans. J Exp Med. 2011;208(13):2581-90.

10.

Paraschos MD, Patrani M, Pistiki A, Katsenos C, Tsaganos T, Netea MG, Giamarellos-Bourboulis EJ, Mandragos K. Defective cytokine production early after multiple traumas: Modulation in severe sepsis. Cytokine. 2015;76(2):222-6.

11.

Spruijt NE, Visser T, Leenen LP. A systematic review of randomized controlled trials exploring the effect of immunomodulative interventions on infection, organ failure, and mortality in trauma patients. Crit Care. 2010;14(4):R150.

12.

Wutzler S, Lustenberger T, Relja B, Lehnert M, Marzi I. [Pathophysiology of multiple trauma: intensive care medicine and timing of treatment]. Chirurg. 2013;84(9):753-8.

13.

Wafaisade A, Lefering R, Bouillon B, Sakka SG, Thamm OC, Paffrath T, Neugebauer E, Maegele M, Trauma TRGS. Epidemiology and risk factors of sepsis after multiple trauma: An analysis of 29,829 patients from the Trauma Registry of the German Society for Trauma Surgery. Crit Care Med. 2011;39(4):621-8.

Page $14 / 22$ 
14.

Stoecklein VM, Osuka A, Lederer JA. Trauma equals danger-damage control by the immune system. J Leukocyte Biol. 2012;92(3):539-51.

15.

Flohe SB, Flohe S, Schade FU. Invited review: deterioration of the immune system after trauma: signals and cellular mechanisms. Innate Immun. 2008;14(6):333-44.

16.

Baue AE, Durham R, Faist E. Systemic inflammatory response syndrome (SIRS), multiple organ dysfunction syndrome (MODS), multiple organ failure (MOF): are we winning the battle?. Shock. 1998;10(2):79-89.

17.

Singer M, Deutschman CS, Seymour CW, Shankar-Hari M, Annane D, Bauer M, Bellomo R, Bernard GR, Chiche JD, Coopersmith CM, et al. The Third International Consensus Definitions for Sepsis and Septic Shock (Sepsis-3). JAMA. 2016;315(8):801-10.

18.

Celikbilek M, Dogan S, Ozbakir O, Zararsiz G, Kucuk H, Gursoy S, Yurci A, Guven K, Yucesoy M. Neutrophil-Lymphocyte Ratio as a Predictor of Disease Severity in Ulcerative Colitis. J Clin Lab Anal. 2013;27(1):72-6.

19.

Pichler M, Hutterer GC, Stoeckigt C, Chromecki TF, Stojakovic T, Golbeck S, Eberhard K, Gerger A, Mannweiler S, Pummer K, et al. Validation of the pre-treatment neutrophil lymphocyte ratio as a prognostic factor in a large European cohort of renal cell carcinoma patients. Brit J Cancer. 2013;108(4):901-7.

20.

Duchesne JC, Tatum D, Jones G, Davis B, Robledo R, DeMoya M, O'Keeffe T, Ferrada P, Jacome T, Schroll R, et al. Multi-institutional analysis of neutrophil-to-lymphocyte ratio (NLR) in patients with severe hemorrhage: A new mortality predictor value. J Trauma Acute Care Surg. 2017;83(5):888-93.

21.

Park KS, Lee SH, Yun SJ, Ryu S, Kim K. Neutrophil-to-lymphocyte ratio as a feasible prognostic marker for pyogenic liver abscess in the emergency department. Eur J Trauma Emerg S. 2019;45(2):343-51.

22.

Siwicka-Gieroba D, Malodobry K, Biernawska J, Robba C, Bohatyrewicz R, Rola R, Dabrowski W. The Neutrophil/Lymphocyte Count Ratio Predicts Mortality in Severe Traumatic Brain Injury Patients. J Clin Med 2019, 8(9).

23.

Chen WQ, Yang JH, Li BB, Peng GY, Li TF, Li LJ, Wang SS. Neutrophil to Lymphocyte Ratio as a Novel Predictor of Outcome in Patients With Severe Traumatic Brain Injury. J Head Trauma Rehab. 2018;33(1):E53-9.

24.

Ear T, McDonald PP. Cytokine generation, promoter activation, and oxidant-independent NF-kappaB activation in a transfectable human neutrophilic cellular model. BMC Immunol. 2008;9:14.

25.

Liao YL, Liu P, Guo FY, Zhang ZY, Zhang ZR. Oxidative Burst of Circulating Neutrophils Following Traumatic Brain Injury in Human. Plos One 2013, 8(7).

26.

Pillay J, Kamp VM, van Hoffen E, Visser T, Tak T, Lammers JW, Ulfman LH, Leenen LP, Pickkers P, Koenderman L. A subset of neutrophils in human systemic inflammation inhibits T cell responses through Mac-1. J Clin Invest. 2012;122(1):327-36.

27.

Hotchkiss RS, Monneret G, Payen D. Sepsis-induced immunosuppression: from cellular dysfunctions to immunotherapy. Nat Rev Immunol. 2013;13(12):862-74.

28.

Heffernan DS, Monaghan SF, Thakkar RK, Machan JT, Cioffi WG, Ayala A. Failure to normalize lymphopenia following trauma is associated with increased mortality, independent of the leukocytosis pattern. Crit Care 2012, 16(1).

29.

Venet F, Davin F, Guignant C, Larue A, Cazalis MA, Darbon R, Allombert C, Mougin B, Malcus C, Poitevin-Later F, et al. Early Assessment of Leukocyte Alterations at Diagnosis of Septic Shock. Shock. 2010;34(4):358-63. 
30.

Gouel-Cheron A, Venet F, Allaouchiche B, Monneret G. CD4 + T-lymphocyte alterations in trauma patients. Crit Care. 2012;16(3):432. 31.

Menges T, Engel J, Welters I, Wagner RM, Little S, Ruwoldt R, Wollbrueck M, Hempelmann G. Changes in blood lymphocyte populations after multiple trauma: Association with posttraumatic complications. Crit Care Med. 1999;27(4):733-40.

32.

Drewry AM, Samra N, Skrupky LP, Fuller BM, Compton SM, Hotchkiss RS. Persistent lymphopenia after diagnosis of sepsis predicts mortality. Shock. 2014;42(5):383-91.

33.

Girardot T, Rimmele T, Venet F, Monneret G. Apoptosis-induced lymphopenia in sepsis and other severe injuries. Apoptosis. 2017;22(2):295-305.

34.

Adrie C, Lugosi M, Sonneville R, Souweine B, Ruckly S, Cartier JC, Garrouste-Orgeas M, Schwebel C, Timsit JF. Persistent lymphopenia is a risk factor for ICU-acquired infections and for death in ICU patients with sustained hypotension at admission. Ann intensive care. 2017;7(1):30.

35.

Chung KP, Chang HT, Lo SC, Chang LY, Lin SY, Cheng A, Huang YT, Chen CC, Lee MR, Chen YJ, et al. Severe Lymphopenia Is Associated with Elevated Plasma Interleukin-15 Levels and Increased Mortality during Severe Sepsis. Shock. 2015;43(6):569-75. 36.

van Dissel JT, van Langevelde P, Westendorp RG, Kwappenberg K, Frolich M. Anti-inflammatory cytokine profile and mortality in febrile patients. Lancet. 1998;351(9107):950-3.

37.

Cavaillon JM, Adib-Conquy M. Bench-to-bedside review: endotoxin tolerance as a model of leukocyte reprogramming in sepsis. Crit Care. 2006;10(5):233.

38.

Hynninen M, Pettila V, Takkunen O, Orko R, Jansson SE, Kuusela P, Renkonen R, Valtonen M. Predictive value of monocyte histocompatibility leukocyte antigen-DR expression and plasma interleukin-4 and - 10 levels in critically ill patients with sepsis. Shock. 2003;20(1):1-4.

39.

Lukaszewicz AC, Grienay M, Resche-Rigon M, Pirracchio R, Faivre V, Boval B, Payen D. Monocytic HLA-DR expression in intensive care patients: interest for prognosis and secondary infection prediction. Crit Care Med. 2009;37(10):2746-52.

40.

Cheron A, Floccard B, Allaouchiche B, Guignant C, Poitevin F, Malcus C, Crozon J, Faure A, Guillaume C, Marcotte G, et al: Lack of recovery in monocyte human leukocyte antigen-DR expression is independently associated with the development of sepsis after major trauma. Crit Care 2010, 14(6).

41.

Landelle C, Lepape A, Voirin N, Tognet E, Venet F, Bohe J, Vanhems P, Monneret G. Low monocyte human leukocyte antigen-DR is independently associated with nosocomial infections after septic shock. Intens Care Med. 2010;36(11):1859-66.

42.

Djordjevic D, Rondovic G, Surbatovic M, Stanojevic I, Udovicic I, Andjelic T, Zeba S, Milosavljevic S, Stankovic N, Abazovic D, et al: Neutrophil-to-Lymphocyte Ratio, Monocyte-to-Lymphocyte Ratio, Platelet-to-Lymphocyte Ratio, and Mean Platelet Volume-toPlatelet Count Ratio as Biomarkers in Critically III and Injured Patients: Which Ratio to Choose to Predict Outcome and Nature of Bacteremia? Mediat inflamm 2018, 2018:3758068.

43.

Chung H, Lee JH, Jo YH, Hwang JE, Kim J. Circulating Monocyte Counts and its Impact on Outcomes in Patients With Severe Sepsis Including Septic Shock. Shock. 2019;51(4):423-9.

44.

Dellinger EP, Anaya DA. Infectious and immunologic consequences of blood transfusion. Crit Care. 2004;8(Suppl 2):18-23. 45. 
Garraud O, Hamzeh-Cognasse H, Pozzetto B, Cavaillon JM, Cognasse F. Bench-to-bedside review: Platelets and active immune functions - new clues for immunopathology? Crit Care. 2013;17(4):236.

46.

Hart S, Cserti-Gazdewich CM, McCluskey SA. Red cell transfusion and the immune system. Anaesthesia. 2015;70(Suppl 1):38-45. e13-36.

\section{Figures}

\section{Figure 1}

$\square 16 \leqslant$ ISS $\leqslant 24 \square 25 \leqslant$ ISS $\leqslant 74$
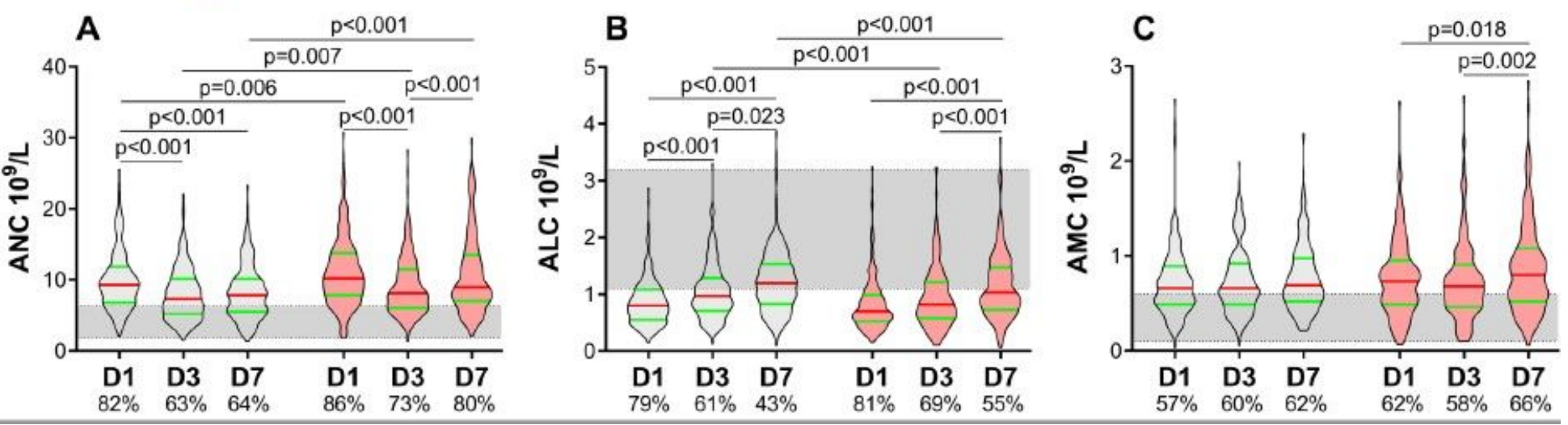

\section{Figure 1}

Patients suffering from more severe trauma have worse alterations in circulating immune cell counts. ANC (absolute neutrophil count) and AMC (absolute monocyte count) rise and ALC (absolute lymphocyte count) decline are common. The proportions of patients with ANC and AMC above the upper limit of the normal range (shaded area) are shown on the x-axis (A and C). The proportions of patients with ALC below the lower limit of the normal range (shaded area) are shown on the $x$-axis $(B)$. ISS $=$ Injury Severity Score; $\mathrm{D} 1$ = day $1 ; \mathrm{D} 3=$ day $3 ; \mathrm{D} 7$ = day 7. 


\section{Figure 2}

$\square$ Non-nosocomial infection $\square$ Nosocomial infection
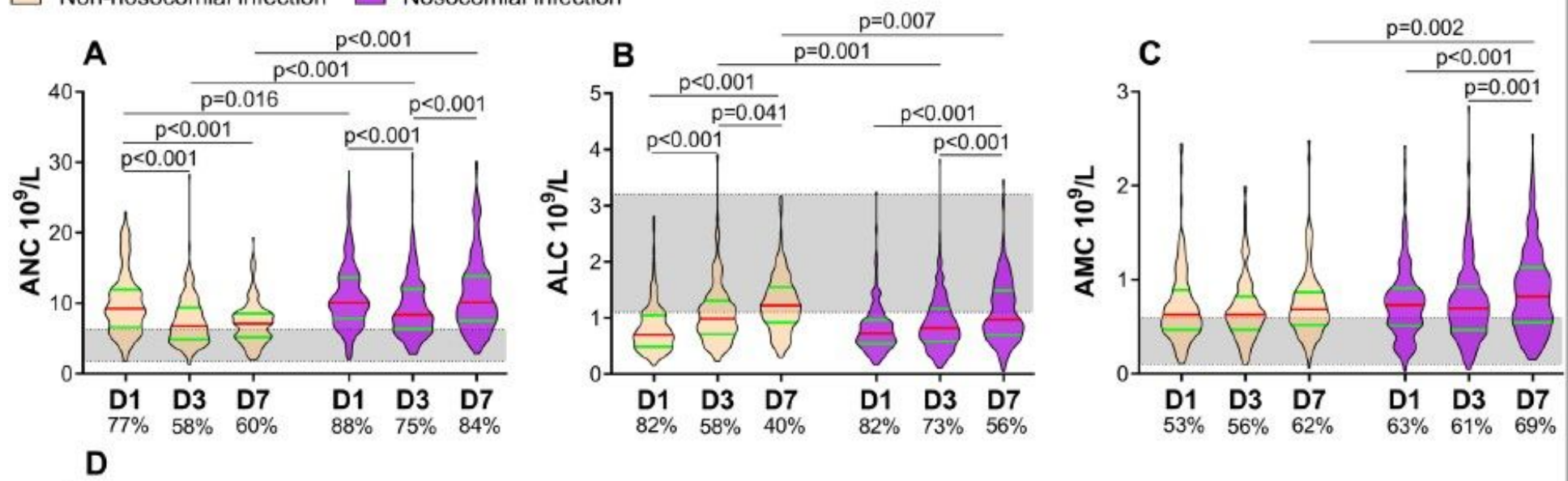

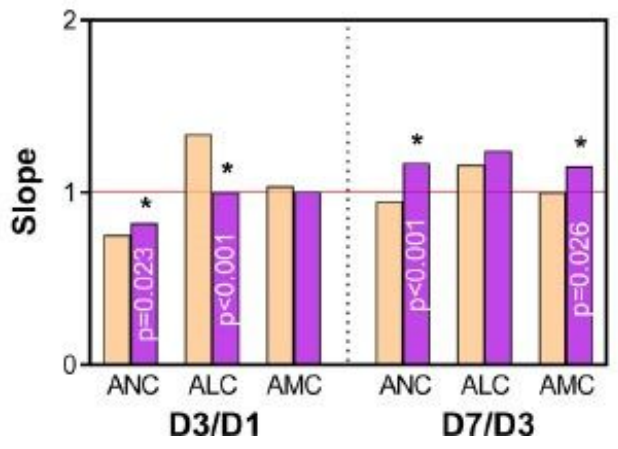

Figure 2

Patients who later developed nosocomial infection have worse alterations of circulating immune cell counts in the early stage. The proportions of patients with ANC (absolute neutrophil count) and AMC (absolute monocyte count) above the upper limit of the normal range (shaded area) are shown on the $x$-axis (A and C). The proportions of patients with ALC (absolute lymphocyte count) below the lower limit of the normal range (shaded area) are shown on the x-axis (B). A value of slope greater than 1 (red line) indicates an upward trend in immune cell counts, and a value less than 1 indicates a downward trend; * Represents a statistical difference between non-nosocomial infection and nosocomial infection groups, and the corresponding p-value is shown on the column (D). D1 = day $1 ; \mathrm{D} 3=$ day $3 ; \mathrm{D} 7=$ day 7 . 
Figure 3
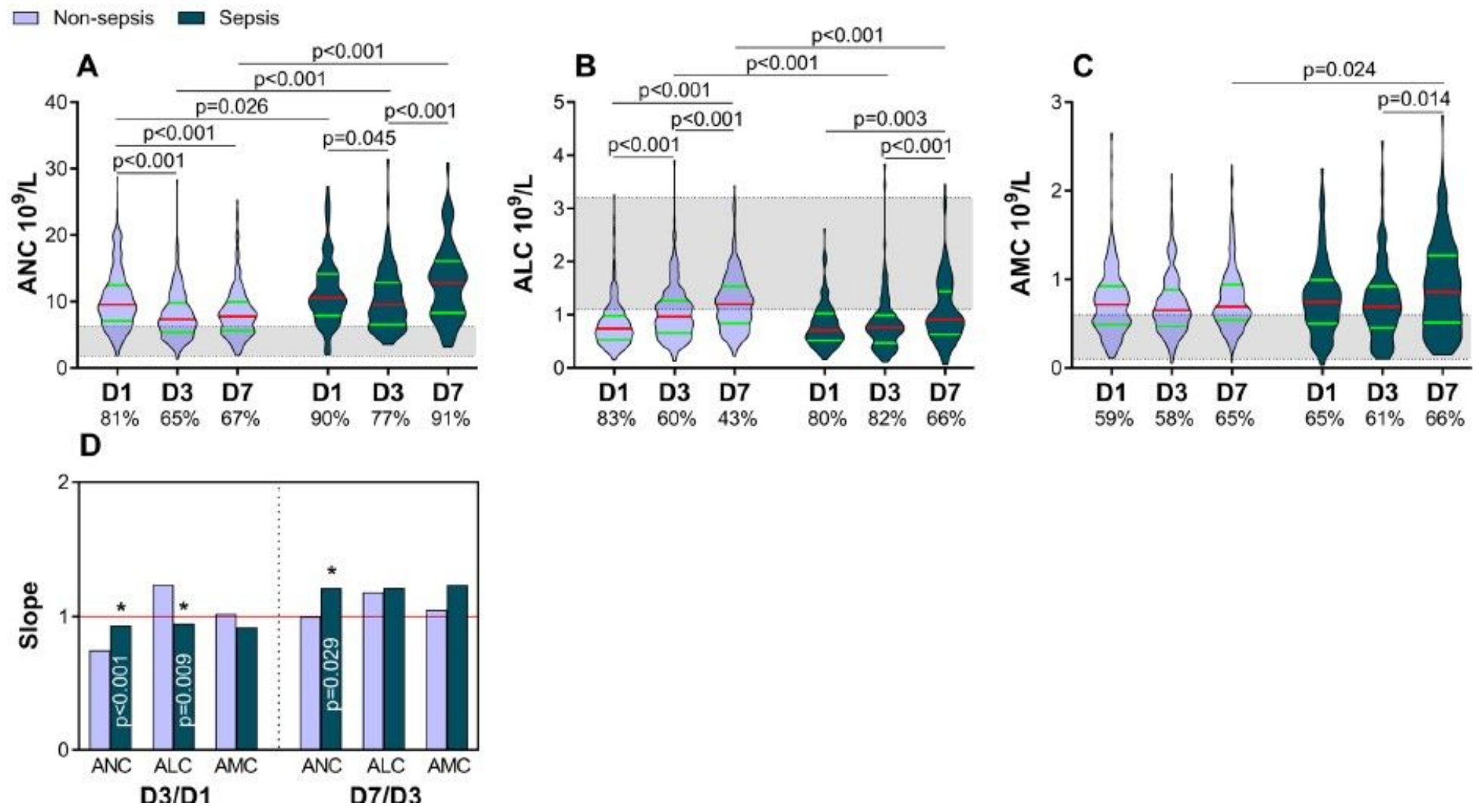

Figure 3

Patients who later developed sepsis have worse alterations of circulating immune cell counts in the early stage. The proportions of patients with ANC (absolute neutrophil count) and AMC (absolute monocyte count) above the upper limit of the normal range (shaded area) are shown on the $x$-axis ( $A$ and $C$ ). The proportions of patients with ALC (absolute lymphocyte count) below the lower limit of the normal range (shaded area) are shown on the $\mathrm{x}$-axis (B). A value of slope greater than 1 (red line) indicates an upward trend in immune cell counts, and a value less than 1 indicates a downward trend; * Represents a statistical difference between non-sepsis and sepsis groups, and the corresponding p-value is shown on the column (D). D1 = day $1 ;$ D3 = day $3 ;$ D7 = day 7 . 
$\square$ Alive Death $\square$ Death due to sepsis

Death due to trauma
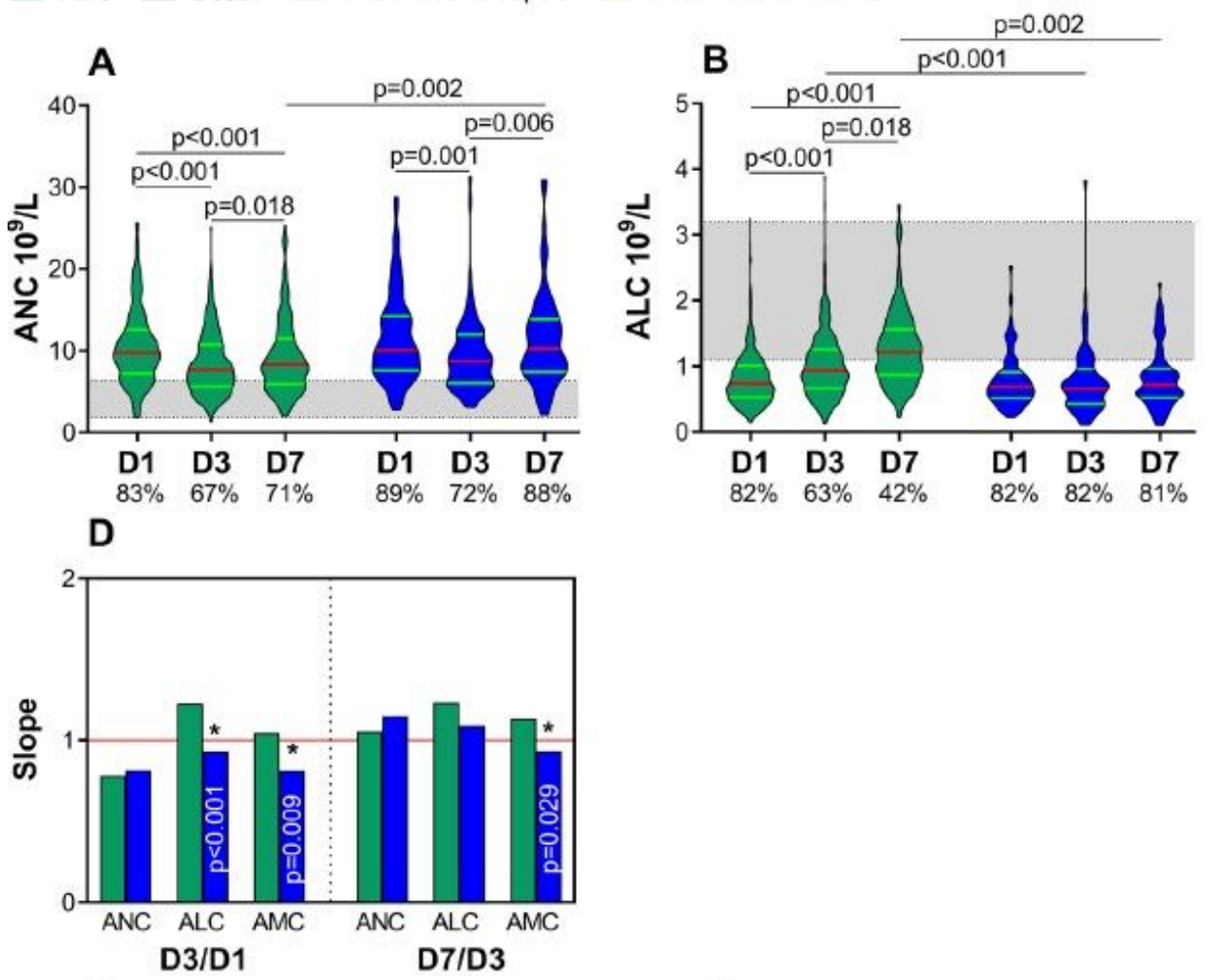

E

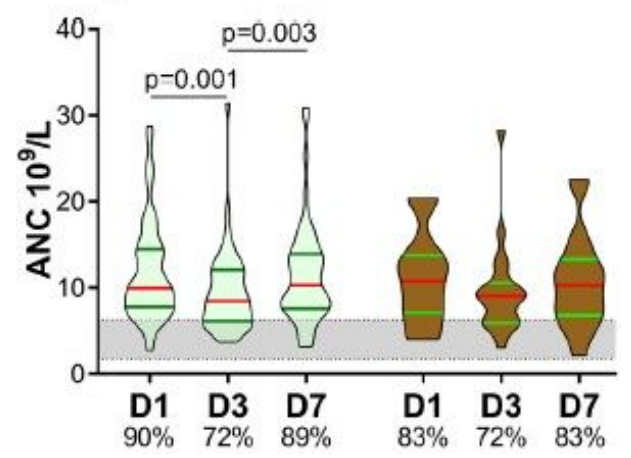

$\mathbf{F}$

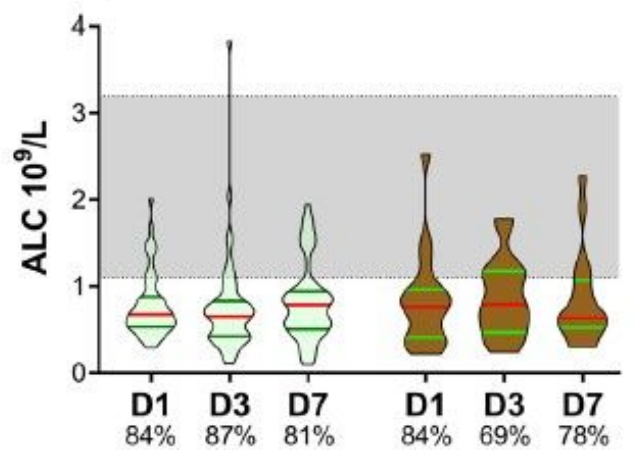

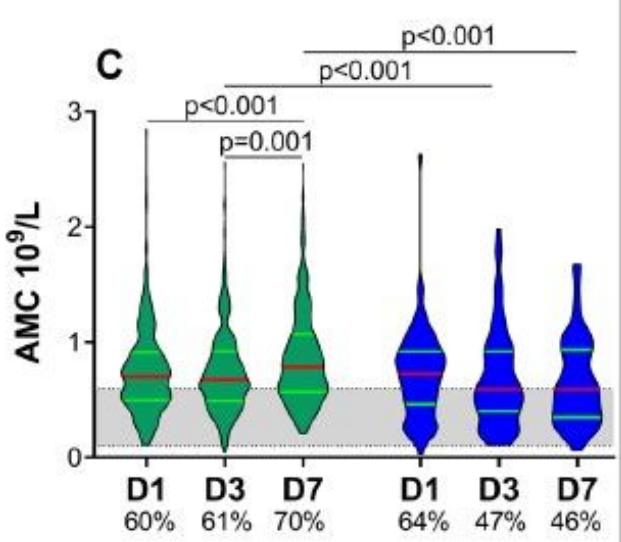

G

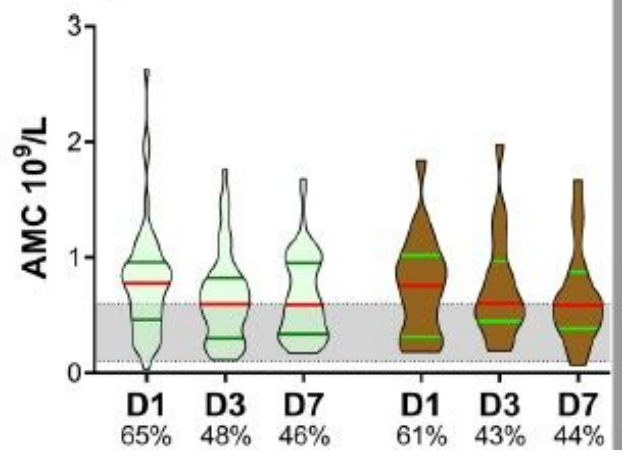

Figure 4

Early circulating immune cell counts show worse alterations in non-survivors, but there is no difference between non-survivors who died of sepsis and trauma. The proportions of patients with ANC (absolute neutrophil count) and AMC (absolute monocyte count) above the upper limit of the normal range (shaded area) are shown on the x-axis (A, C, E and G). The proportions of patients with ALC (absolute lymphocyte count) below the lower limit of the normal range (shaded area) are shown on the $x$-axis (B and $F)$. A value of slope greater than 1 (red line) indicates an upward trend in immune cell counts, and a value less than 1 indicates a downward trend; * Represents a statistical difference between alive and death groups, and the corresponding $p$-value is shown on the column (D). D1 = day 1; D3 = day 3; D7 = day 7. 
Figure 5

A

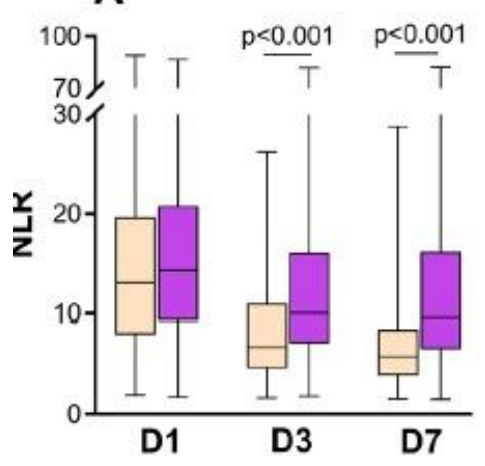

B

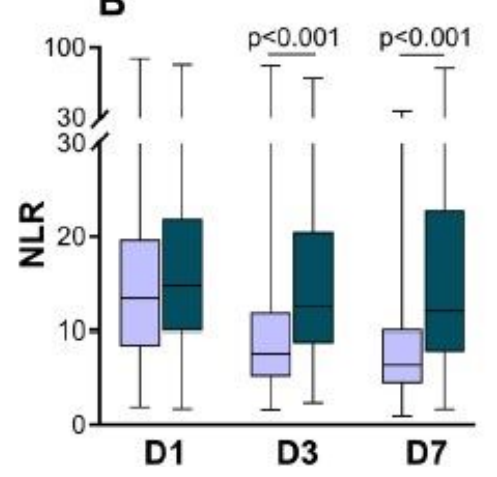

C

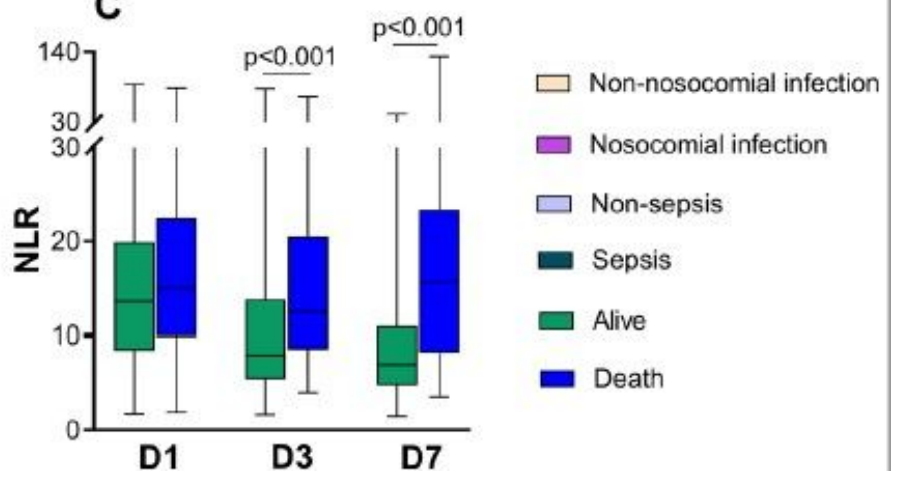

Figure 5

Differences in NLR (neutrophil-lymphocyte ratio) were compared between non-nosocomial infection and nosocomial infection groups (A), non-sepsis and sepsis groups (B), and alive and death groups (C) on day 1 (D1), day 3 (D3) and day 7 (D7).

\section{Figure 6}

\section{A}

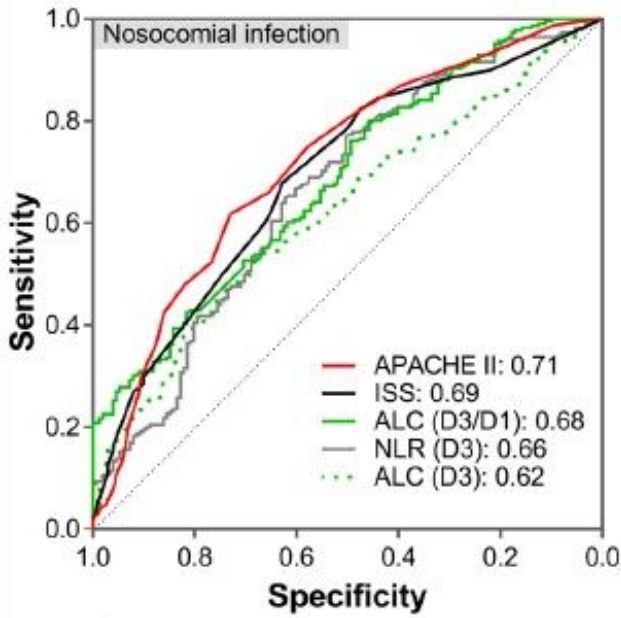

D

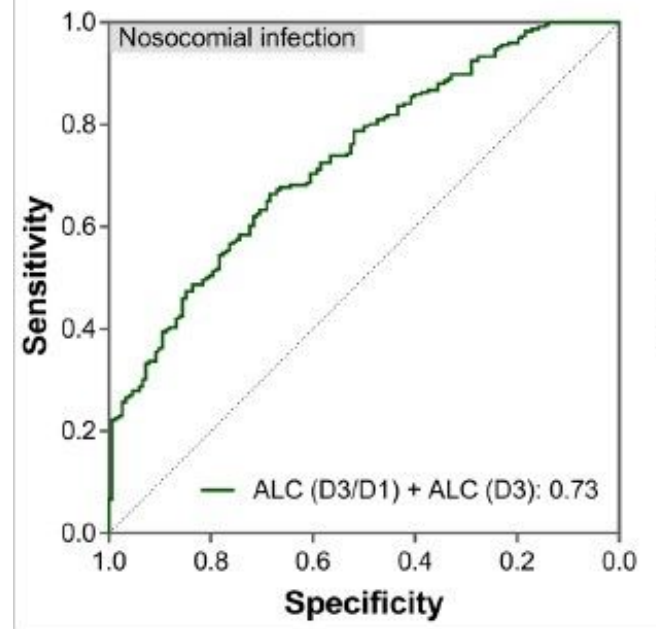

B

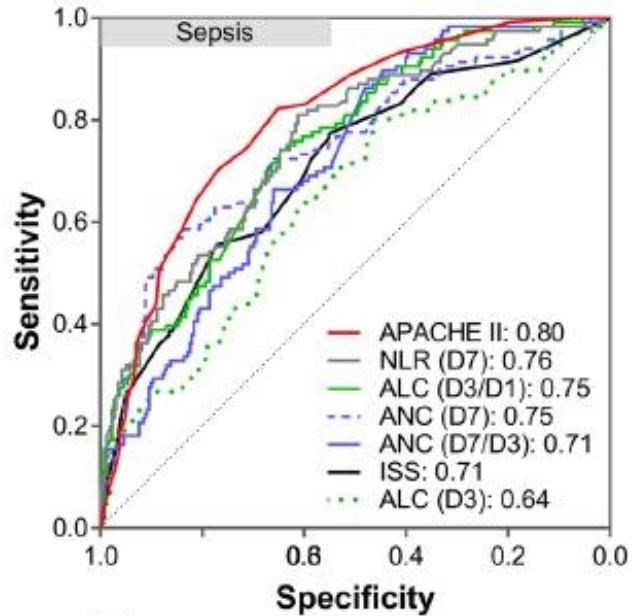

E

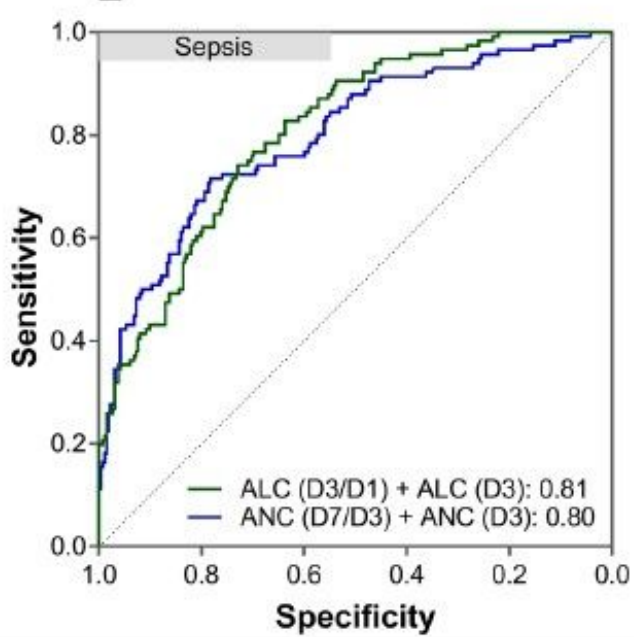

C

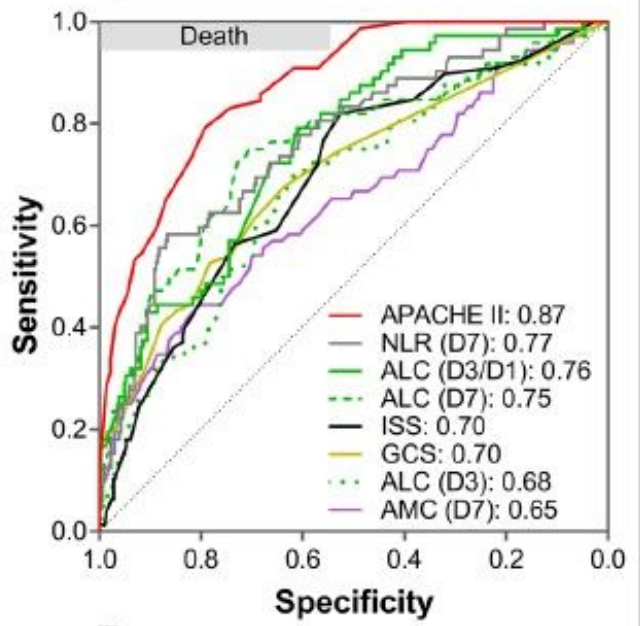

$\mathbf{F}$

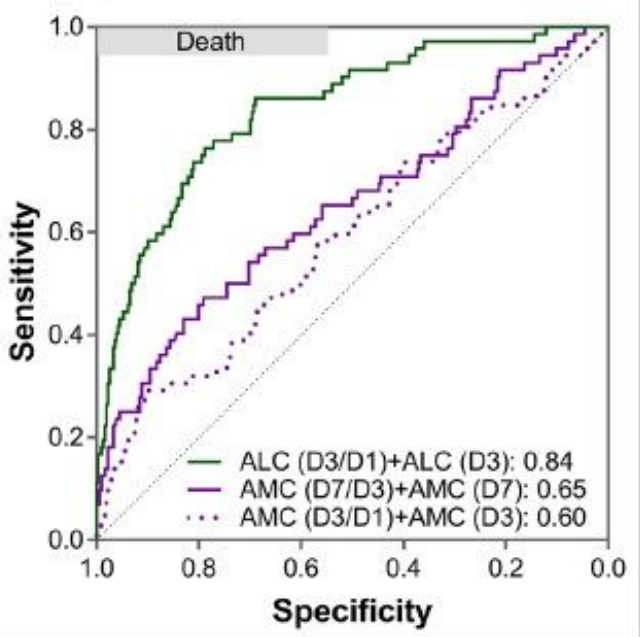

\section{Figure 6}

Receiver operating characteristic (ROC) curve analysis to compare the values of indicators in predicting the risk of nosocomial infection, sepsis and mortality. The area under the ROC curve of each indicator is presented in the figure. APACHE II = Acute Physiology and Chronic Health Evaluation II score; ISS = Injury Severity Score; GCS = Glasgow coma scale; ANC = absolute 
neutrophil count; $\mathrm{ALC}=$ absolute lymphocyte count; $\mathrm{AMC}=$ absolute monocyte count; $\mathrm{D} 1=$ day $1 ; \mathrm{D} 3=$ day $3 ; \mathrm{D} 7=$ day $7 ; \mathrm{D} 3 / \mathrm{D} 1=$ day $3 /$ day $1 ; D 7 / D 3=$ day $7 /$ day 3 .

\section{Supplementary Files}

This is a list of supplementary files associated with this preprint. Click to download.

- Additionalfile1.xlsx 